\title{
ENUMERATION OF THE LICHEN-FORMING AND LICHENICOLOUS FUNGI OF THE CĂLIMANI MOUNTAINS (EASTERN CARPATHIANS, ROMANIA)
}

\section{László LőKös ${ }^{1}$, Florin CRIŞAN ${ }^{2}$, Jae-Seoun HUR ${ }^{3}$, Nóra VARGA ${ }^{4}$ and Edit FARKAS ${ }^{4}$}

\author{
${ }^{1}$ Department of Botany, Hungarian Natural History Museum, \\ H-1431 Budapest, Pf. 137, Hungary; lokos.laszlo@nhmus.hu \\ ${ }^{2}$ Department of Taxonomy and Ecology, Faculty of Biology and Geology, \\ Babeş-Bolyai University, Cluj, Romania \\ ${ }^{3}$ Korean Lichen Research Institute, Sunchon National University, Suncheon, Republic of Korea \\ ${ }^{4}$ Institute of Ecology and Botany, MTA Centre for Ecological Research, Hungarian Academy of Sciences, \\ H-2163 Vácrátót, Alkotmány utca 2-4, Hungary
}

Lőkös, L., Crişan, F., Hur, J.-S., Varga, N. \& Farkas, E. (2018): Enumeration of the lichen-forming and lichenicolous fungi of the Călimani Mountains (Eastern Carpathians, Romania). - Studia bot. bung. 49(1): 5-40.

\begin{abstract}
Having studied ca 300 specimens and 23 publications, 170 taxa of lichen-forming and lichenicolous fungi are reported from various sources, 140 from published literature records and old herbarium specimens, and 30 species from recently collected material. One lichen species (Xylographa pallens), and two lichenicolous fungi (Clypeococcum hypocenomycis, Endococcus macrosporus) are new for Romania, and 30 lichen species are considered as new for the Călimani Mountains. According to available specimens, Coniocybe gracilenta was revised as Chaenotheca furfuracea, and Peltigera aphthosa as P. didactyla.
\end{abstract}

Key words: Călimani Mts, Eastern Carpathians, lichen-forming fungi, lichenicolous fungi, Romania

\section{INTRODUCTION}

Exploration of the lichen flora of the Călimani Mts (Eastern Carpathians, Romania) has received less attention from lichenologists in the past, comparing with other high mountain areas. Although lichen specimens were collected in the Călimani Mts by Nyárády E. Gy. as early as 1914, the first lichen records of 44 species were published by Paul Cretzoiu between 1931 and 1943 from various localities in the Călimani Mts in several papers (Cretziou 1931, 1935, 1936a, b, 1937, 1940, 1941, 1943a, $b$, Cretziou and Klement 1935, Servit and Cretziou 1936). Vasile Codoreanu visited the main peaks and the valley 'Drâglele' in July 1949 and 1950, and reported 74 taxa in CODOREANU $(1952,1954)$ ( 55 new to the lichen flora of the mountains among them). All of these records (96 taxa) were 
summarised in the first Romanian lichen catalogue by MORUzi et al. (1967) with nomenclatural revisions in some cases, but without any taxonomic revisions. However, one formerly published species was omitted from their catalogue, and four records were mistakenly reported from the Călimani Mts.

Considerable contributions were made also by Andrei Stoie and Florin Crişan who published 73 foliose and fruticose lichen species from some western areas of the Călimani Mts (Reservația Bistricioru, Rezervația Pădurea din Şes, Rezervația Valea Repedea,) with 47 new floristical records (Stole 1999, 2001, STOIE and CRIŞAN 1988). Unfortunately these latter results were not included in Maria Ciurchea's Romanian lichen catalogue (Ciurchea 2004). However, Ciurchea made considerable nomenclatural and also taxonomic revisions, and finally 96 species were listed from the Călimani Mts in her catalogue.

Several species should be excluded due to erroneous citations, nomenclatural mistakes or misidentifications, therefore the number of species published from the Călimani Mts is 128 altogether. Specimens collected and/or published from the nearby Poiana Stampei, Dornişoara and surroundings were considered to belong to the Bârgăului Mts, and were omitted from this enumeration so far.

Further revisions on the available old specimens are also necessary and it is in progress. Unfortunately, most of the specimens are not available any more. As a result of direct searching for voucher specimens in CL almost all specimens published in CODOREANu (1952) could be found, but no any specimens published in CODOREANU (1954). Another difficulty with the available voucher specimens is that no exact locality data are given in many cases.

The scientific results of an international, short-time, lichenological field study carried out by F. Crişan, J.-S. Hur and L. Lőkös in 2009 at the northern parts of the Călimani Mts are also discussed here.

The aims of this paper are 1) to give an account on the history of the lichenological exploration in the Călimani Mts; 2) to prepare a checklist as complete as possible including the results of our preliminary investigations in 2009, with own revisions; 3 ) to confirm all old records by fresh collections with exact localities and coordinates.

\section{MATERIAL AND METHODS}

Voucher specimens are deposited in BP (90 specimens), KoLRI (Korean Lichen Research Institute, Suncheon, 87 specimens) and hb. Crişan ( 53 specimens). Most specimens of V. Codoreanu and some of P. Cretzoiu are deposited in CL (63 specimens) (abbreviations follow the Index Herbariorum online; THIERs 2018).

Locality data for the recent collections were measured by Garmin GPS devices (WGS84). The locality map (Fig. 1) was prepared by the computer program 
for geographical information system, Quantum GIS (QGIS 2.18 Las Palmas, 2018) based on the Central European grid system of $5 \mathrm{~km} \times 6 \mathrm{~km}$ units (BORHIDI 1984, Ni KLFELD 1971).

Morphological-anatomical investigations and spot tests (ORANGE et al. 2010) were carried out by standard methods using Olympus SZX-7 binocular dissecting microscope and Olympus CX-41 stereomicroscope. Micrographs were prepared by Olympus E450 camera (with Quick Photo Camera 2.3 software). For the identification of chemical substances of critical taxa, standard HPTLC methods with solvent system C were carried out (ARUP et al. 1993). For identifications, mainly SMITH et al. (2009) and WirTH et al. (2013) were used.

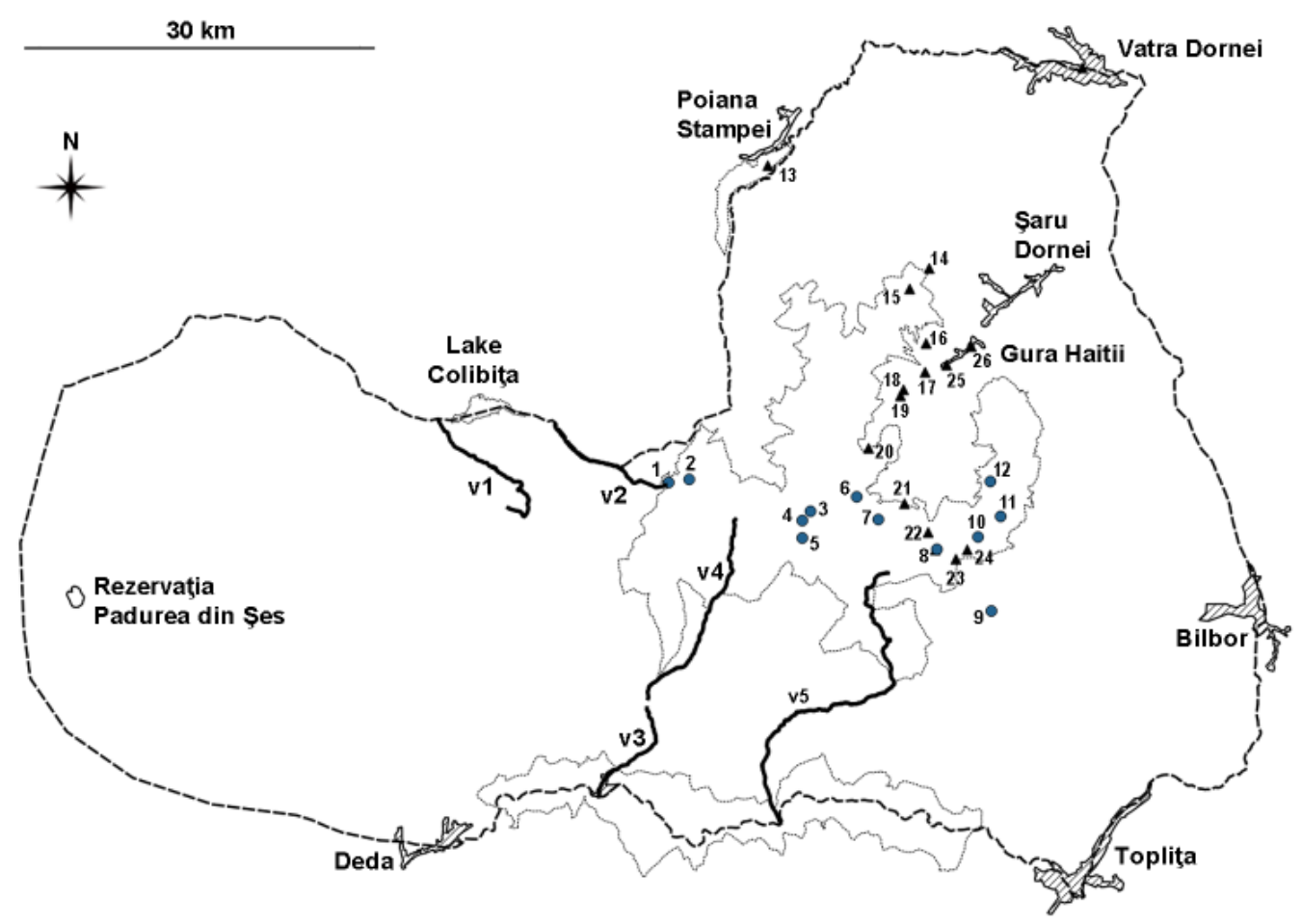

Fig. 1. Collecting localities in the Călimani Mts, Eastern Carpathians, Romania (dashed line = border of the Călimani Mts; dotted line = border of the Călimani National Park; circles = localities from former sources; triangles = localities from the fieldwork in 2009. - $1=$ Mt Zurzugău; $2=\mathrm{Mt}$ Bistricioru; 3 = Mt Gruiu; $4=$ Mt Ruschi, $5=$ Mt Tihu; $6=$ Mt Pietrosul; 7 = Mt Negoiu Unguresc; 8 = Mt Retițiş; $9=$ Mt Puturosul; $10=$ Mt Bradul Ciont; $11=$ Mt Voivodeasa; $12=$ Mt Căliman Izvor; 13 = Poiana Stampei; 14 = Cerbul Monastery, 15 = Mt 12 Apostols; 16 = Porțile Hârlei; 17-20 = valley of Tarnița stream; 21-22 = Mt Negoi (near the sulphur mine); $23=$ Lake Iezer; $24=$ near Refuge Salvamont, 25-26 = Gura Haitii (valley of Neagra Şarului river); v1 = Repedea valley (Rezervaţia Valea Repedea); v2 = Colbu valley (Rezervația Bistricioru); v3 = Răstolnița valley; v4 = Tihu valley; v5 = Draglele valley (?)). 
Nomenclature follows mostly Index Fungorum (CABI 2018) and MycoBank (ROBERT et al. 2018).

\section{RESULTS}

\section{Enumeration}

Taxa are arranged alphabetically in the following enumeration, records within taxa are arranged chronologically. Records - closed by a full stop - are separated by hyphens. In case of literature records the original text of the first source is given followed by the adequate references in brackets. In case of herbarium specimens the label text is cited (with additional habitat information from literature in brackets) followed by the collector and date of collecting (if available), the herbarium information in square brackets and all concerning references (in brackets).

Species new for Romania are marked by double exclamation mark (!!), species new for Călimani Mts are marked by a single exclamation sign (!), and lichenicolous species are marked by an asterisk $\left({ }^{*}\right)$.

Alectoria ochroleuca (Schrank) A. Massal. - Distr. Mureş: Munții Călimani, 1,900-2,300 m s. m., Pietrosul. Coll.: Nyárády, E. I., 13.08.1936 (CREtzoiu 1939, Moruzi et al. 1967, Ciurchea 2004). - M-ții Călimanului, vf. Bradul Ciont, 1,800 m. alt. Coll.: Codoreanu, V., 07.1949 [CL] (Codoreanu 1952, Moruzi et al. 1967, Ciurchea 2004). - Rezervația Valea Repedea: near the spring of stream 'Repedele', on a dry spruce branch, 21.07.1997 [hb. Crişan 1204] (STOIE and CRIşAN 1998). - Romania, Călimani, Vf Rețitiş, on soil. Lat.: $47^{\circ} 05.847^{\prime}$ N; Long.: $25^{\circ} 14.737^{\prime}$ E, Alt.: 2,022 m a.s.l. Leg.: Hur, J.-S. (RO-09020), 25.06.2009 [KoLRI 12980]. - Romania, Suceava County, Călimani Mts, Călimani National Park, Mt Rețitiş, near the meteorological station (Stația Meteo), ca $11 \mathrm{~km} \mathrm{SSW} \mathrm{of}$ Gura Haitii, on siliceous rock. Lat.: $47^{\circ} 05^{\prime} 51.1^{\prime \prime} \mathrm{N}$; Long.: $25^{\circ} 14^{\prime} 46.1^{\prime \prime} \mathrm{E}$; Alt.: 2,006 m a.s.l. Leg.: Lőkös, L., 25.06.2009 [BP 93341].

Alectoria sarmentosa (Ach.) Ach. - Distr. Mureş: mts Călimani, dans la Valea Tihului, alt. 780 m., sur Fagus sylvatica (Cretzoiv 1937, 1941, Moruzi et al. 1967, CiurcheA 2004).

Anaptychia ciliaris (L.) A. Massal. - Rezervația Pădurea din Şes, on oak bark, 04.08.1996 [hb. Crişan 787] (STOIE and CRIŞAN 1998).

! Arthrorhaphis alpina (Schaer.) R. Sant. - Romania, Călimani, Reservation Pinus Cembra, on moss. Lat.: $47^{\circ} 06.381^{\prime} \mathrm{N}$; Long.: $25^{\circ} 14.411^{\prime} \mathrm{E}$, Alt.: 1,653 m a.s.l. Leg.: Hur, J.-S. (RO-09088), 28.06.2009 [KoLRI 13040 as A. citrinella]. Romania, Suceava County, Călimani Mts, Călimani National Park, Mt Rețitiş, 
near the meteorological station (Stația Meteo), ca $11 \mathrm{~km} \mathrm{SSW}$ of Gura Haitii, on acid soil. Lat.: $47^{\circ} 05^{\prime} 51.1$ " N; Long.: $25^{\circ} 14^{\prime} 46.1$ " E; Alt.: $2,006 \mathrm{~m}$ a.s.l. Leg.: Lőkös, L., 25.06.2009 [BP 93331].

Bacidia caesiomarginata (Kernst.) Lettau - M-ții Călimanului, aflat pe muşchi pe m-tele Puturosul, la 1,150 m alt. (Codoreanu 1954, Moruzi et al. 1967, Ciurchea 2004).

! Baeomyces placophyllus Ach. - Romania, Suceava County, Călimani Mts, Călimani National Park, along tourist path between Stâncile Lucaciu and Stâncile Doisprezece Apostoli, on acid soil. Lat.: 47 $13^{\prime} 27.9^{\prime \prime} \mathrm{N}$; Long.: $25^{\circ} 12^{\prime} 41.5^{\prime \prime} \mathrm{E}$; Alt.: 1,726 m a.s.l. Leg.: Lőkös, L., 27.06.2009 [BP 93488].

Baeomyces rufus (Huds.) Rebent. - M-ții Călimanului, aflat pe pământ pe m-tele Puturosul, $1200 \mathrm{~m}$ alt. (Codoreanu 1954 as B. callianthus, Moruzi et al. 1967 as B. callianthus, Ciurchea 2004). - Romania, Călimani, Apostoli (Gura Haitii), on rock. Lat.: $47^{\circ} 14.201^{\prime} \mathrm{N}$; Long.: $25^{\circ} 14.223^{\prime} \mathrm{E}$, Alt.: 1,637 m a.s.l. Leg.: Hur, J.-S. (RO-09055), 27.06.2009 [KoLRI 13013]. - Romania, Călimani, Reservation Pinus Cembra, on moss. Lat.: $47^{\circ} 06.379^{\prime}$ N; Long.: $25^{\circ} 14.430^{\prime} \mathrm{E}$, Alt.: 1,650 m a.s.l. Leg.: Hur, J.-S. (RO-09095), 28.06.2009 [KoLRI 13045]. - Romania, Călimani, Tarnița (Gura Haitii), on rock. Lat.: $47^{\circ} 08.980^{\prime} \mathrm{N}$; Long.: $25^{\circ}$ 11.640' E, Alt.: 1,377 m a.s.l. Leg.: Hur, J.-S. (RO-09045, RO-09046), 26.06.2009 [KoLRI 13004, KoLRI 13005]. - Romania, Suceava County, Călimani Mts, Mt Negoi, near the sulphur mine (Exploatarea Călimani), ca $9 \mathrm{~km} \mathrm{SSW}$ of Gura Haitii, on acid soil. Lat.: $47^{\circ} 07^{\prime} 17.3^{\prime \prime} \mathrm{N}$; Long.: $25^{\circ} 13^{\prime} 20.0^{\prime \prime}$ E; Alt.: 1,673 m a.s.l. Leg.: Lőkös, L., 25.06.2009 [BP 93328]. - Romania, Suceava County, Călimani Mts, Călimani National Park, Mt Rețitiş, near the meteorological station (Staţia Meteo), ca $11 \mathrm{~km} \mathrm{SSW}$ of Gura Haitii, on acid soil. Lat.: $47^{\circ} 05^{\prime} 51.1^{\prime \prime} \mathrm{N}$; Long.: $25^{\circ} 14$ ' 46.1" E; Alt.: 2,006 m a.s.l. Leg.: Lőkös, L., 25.06.2009 [BP 93332] - M-ții Călimani, Parcul Național Călimani, Vf Rețitiş near the meteorological station, on acid soil, 25.06.2009 [hb. Crişan 2201]..

Bellemerea cinereorufescens (Ach.) Clauzade et Cl. Roux - Distr. Mureş: Munții Călimani în Valea Tihului, 780 m. s. m., pe roce eruptive (CretzorU $1943 a$ as Lecanora cinereorufescens, MORUZI et al. 1967 as L. cinereorufescens, Ciurchea 2004).

! Brianaria sylvicola (Körb.) S. Ekman et M. Svenss. - Romania, Suceava County, Călimani Mts, Călimani National Park, Mt Negoi, near the sulphur mine (Exploatarea Călimani), on siliceous rock. Lat.: $47^{\circ} 06^{\prime} 24.7^{\prime \prime} \mathrm{N}$; Long.: $25^{\circ} 14^{\prime}$ 25.3” E; Alt.: 1,637 m a.s.1. Leg.: Lőkös, L., 28.06.2009 [BP 96311].

! Brodoa intestiniformis (Vill.) Goward - Romania, Călimani, Vf Rețitiş, on rock. Lat.: $47^{\circ} 05.847^{\prime} \mathrm{N}$; Long.: $25^{\circ} 14.737^{\prime} \mathrm{E}$, Alt.: 2,022 m a.s.l. Leg.: Hur, J.-S. (RO-09028), 25.06.2009 [KoLRI 12988]. - Romania, Suceava County, Călimani Mts, Călimani National Park, Mt Rețitiş, near the meteorological station (Stația 
Meteo), ca $11 \mathrm{~km} \mathrm{SSW}$ of Gura Haitii, on siliceous rock. Lat.: $47^{\circ} 05^{\prime} 51.1^{\prime \prime} \mathrm{N}$; Long.: $25^{\circ}$ 14' 46.1" E; Alt.: 2,006 m a.s.l. Leg.: Lőkös, L., 25.06.2009 [BP 93342].

Bryoria bicolor (Ehrh.) Brodo et D. Hawksw. - Rezervația Valea Repedea: on a dry spruce near the peak 'Poiana Calului', 23.07.1997 [hb. Crişan 1221] (STOIE and CRIşAN 1998).

Bryoria capillaris (Ach.) Brodo et D. Hawksw. - Rezervația Bistricioru (STOIE 1999).

Bryoria fuscescens (Gyelnik) Brodo et D. Hawksw. - Rezervația Valea Repedea: near stream 'Repedele', on the dry branches of spruces, 23.07.1997 [hb. Crişan 1219] (STOIE and CRIŞAN 1998). - Rezervația Bistricioru, pârâului Colbu (STOIE 1999).

Bryoria implexa (Hoffm.) Brodo et D. Hawksw. - Distr. Mureş: mts Călimani, sur le mt Tihu et dans la Poiana Tihulet, sur Picea excelsa, altitude $1,500 \mathrm{~m}$. (Cretzoiv 1937 as Alectoria implexa f. fuscidula, Moruzi et al. 1967 as A. implexa f. fuscidula, Ciurchea 2004). - M-ții Călimanului, Stâna Retițiş, 1,400 m alt. Coll.: Codoreanu, V., 28.07.1949 [CL as $A$. implexa] (Codoreanu 1952 as A. implexa, Moruzi et al. 1967 as A. implexa, Ciurchea 2004). - Romania, Călimani, Apostoli (Gura Haitii), on rock. Lat.: 47 $13.453^{\prime}$ N; Long.: $25^{\circ} 12.715^{\prime}$ E, Alt.: 1,740 m a.s.l. Leg.: Hur, J.-S. (RO-09078), 27.06.2009 [KoLRI 12786]. Romania, Călimani, Potica (Gura Haitii), on twig. Lat.: $47^{\circ} 11.445^{\prime}$ N; Long.: $25^{\circ}$ 14.262' E, Alt.: 1,100 m a.s.l. Leg.: Hur, J.-S. (RO-09043), 26.06.2009 [KoLRI 13002]. - Romania, Călimani, Reservation Pinus Cembra, on bark. Lat.: $47^{\circ}$ 06.381' N; Long.: $25^{\circ} 14.411^{\prime}$ E, Alt.: 1,653 m a.s.l. Leg.: Hur, J.-S. (RO-09087), 28.06.2009 [KoLRI 13039]. - Romania, Călimani, Tarnița (Gura Haitii), on twig. Lat.: $47^{\circ} 08.980^{\prime}$ N; Long.: $25^{\circ} 11.640$ ' E, Alt.: 1,377 m a.s.l. Leg.: Hur, J.-S. (RO09044), 26.06.2009 [KoLRI 13003]. - Romania, Călimani, Vf Negoi Romanesc, on bark. Lat.: $47^{\circ} 07.286^{\prime} \mathrm{N}$; Long.: $25^{\circ} 13.348^{\prime} \mathrm{E}$, Alt.: 1,680 m a.s.l. Leg.: Hur, J.-S. (RO-09007), 25.06.2009 [KoLRI 12973].

Bunodophoron melanocarpum (Sw.) Wedin - Distr. Mureş: Călimani-Gebirge, Pietrosul-Gipfel, bei 2,100 m s. m., auf Eruptivgestein (SERVít and CretzOIU 1937 as Sphaerophorus melanocarpus, CRETzOIU 1943a as S. melanocarpus, MORUZi et al. 1967 as S. melanocarpus, CiURCHEA 2004 as S. melanocarpus).

Cetraria ericetorum Opiz - Rezervația Bistricioru, vf. Zurzugău (STOIE 1999). - Romania, Călimani, Apostoli (Gura Haitii), on moss. Lat.: $47^{\circ} 13.453^{\prime} \mathrm{N}$; Long.: $25^{\circ} 12.715^{\prime}$ E, Alt.: 1,740 m a.s.l. Leg.: Hur, J.-S. (RO-09077), 27.06.2009 [KoLRI 13031]. - M-ții Călimani, Parcul Național Călimani, Gura Haitii, on moss, 27.06.2009 [hb. Crişan 2251].

Cetraria islandica (L.) Ach. - Distr. Mureş: munții Călimani, dela vârful Izvor peste Pietrosul la vrf. Ruschi, 1,900-2,102 m s. m. Coll.: Nyárády, E. I., 22.06.1914 (Cretzoiu 1939 as C. islandica f. platyna, Moruzi et al. 1967 
as C. islandica f. platyna, CiurChe A 2004). - Jud. Mureş: munții Călimani pe vîrful Gruiu la ca 1,700 m. pe sol, în massive de Pinus mughus (CretzoiU and Klement 1935). - M-ții Călimanului (Codoreanu 1952, Moruzi et al. 1967, Ciurchea 2004). - Rezervaţia Pădurea din Şes (Stole and Crişan 1998). Rezervația Bistricioru (Store 1999). - Romania, Călimani, Reservation Pinus Cembra, on bark. Lat.: $47^{\circ} 06.400^{\prime} \mathrm{N}$; Long.: $25^{\circ} 14.437^{\prime} \mathrm{E}$, Alt.: $1,648 \mathrm{~m}$ a.s.l. Leg.: Hur, J.-S. (RO-09085), 28.06.2009 [KoLRI 13037]. - Romania, Călimani, Vf Rețitiş, on soil. Lat.: $47^{\circ} 05.847^{\prime} \mathrm{N}$; Long.: $25^{\circ} 14.737^{\prime} \mathrm{E}$, Alt.: $2,022 \mathrm{~m}$ a.s.l. Leg.: Hur, J.-S. (RO-09014), 25.06.2009 [KoLRI 12979]. - Romania, Suceava County, Călimani Mts, Călimani National Park, Mt Rețitiş, near the meteorological station (Stația Meteo), ca $11 \mathrm{~km} \mathrm{SSW}$ of Gura Haitii, on acid soil. Lat.: $47^{\circ}$ 05' 51.1" N; Long.: $25^{\circ} 14$ ' 46.1" E; Alt.: 2,006 m a.s.l. Leg.: Lőkös, L., 25.06.2009 [BP 93334]. - Romania, Suceava County, Călimani Mts, Călimani National Park, along tourist path between Stâncile Lucaciu and Stâncile Doisprezece Apostoli, growing together with Parmeliopsis ambigua and Vulpicida pinastri on bark (Picea abies). Lat.: $47^{\circ} 13^{\prime} 27.9^{\prime \prime} \mathrm{N}$; Long.: $25^{\circ} 12^{\prime} 41.5^{\prime \prime} \mathrm{E}$; Alt.: 1,726 m a.s.l. Leg.: Lőkös, L., 27.06.2009 [BP 96296]. - Romania, Suceava County, Călimani Mts, Călimani National Park, Mt Negoi, near the sulphur mine (Exploatarea Călimani), on bark (Picea abies). Lat.: $47^{\circ} 06^{\prime} 24.7^{\prime \prime} \mathrm{N}$; Long.: $25^{\circ} 14^{\prime} 25.3^{\prime \prime} \mathrm{E}$; Alt.: 1,637 m a.s.l. Leg.: Lőkös, L., 28.06.2009 [BP 96305]. - M-ții Călimani, Parcul Naţional Călimani, Vf. Rețitiş, near the meteorological station, on soil, 25.06.2009 [hb. Crişan 2202].

Cetraria sepincola (Ehrh.) Ach. - Rezervația Bistricioru (Store 1999). Romania, Suceava County, Călimani Mts, Călimani National Park, Mt Rețitiş, near the meteorological station (Stația Meteo), ca $11 \mathrm{~km} \mathrm{SSW}$ of Gura Haitii, on bark (Pinus mugo). Lat.: $47^{\circ} 05^{\prime}$ 51.1" N; Long.: $25^{\circ} 14^{\prime} 46.1$ " E; Alt.: 2,006 m a.s.l. Leg.: Lőkös, L., 25.06.2009 [BP 93350].

! Chaenotheca chrysocephala (Ach.) Th. Fr. - Romania, Suceava County, Călimani Mts, in the valley of the stream Tarnița, on bark (Picea abies). Lat.: $47^{\circ}$ 10’ 49.5” N; Long.: 25 13’ 17.6” E; Alt.: 1,121 m a.s.l. Leg.: Lőkös, L., 26.06.2009 [BP 93366]. - Romania, Suceava County, Călimani Mts, northern side of Vârful Haitii in the valley of the stream Tarnița, on bark (Picea abies). Lat.: $47^{\circ} 09^{\prime} 00.3^{\prime \prime}$ N; Long.: 25 11' 41.4" E; Alt.: 1,341 m a.s.l. Leg.: Lőkös, L., 26.06.2009 [BP 93457]. - Chaenoteca chrysocephala f. filaris (Ach.) Blomb. et Forssell was mistakenly reported by MORUzi et al. (1967) and BARTóK (2002) from the Călimani Mts as 'Mții Călimani, lingă Tuşnad'

! Chaenotheca ferruginea (Turner) Mig. - Romania, Suceava County, Călimani Mts, northern side of Vârful Haitii in the valley of the stream Tarnița, on bark (Picea abies). Lat.: $47^{\circ} 09^{\prime} 00.3$ ” N; Long.: 25 11' 41.4” E; Alt.: 1,341 m a.s.l. Leg.: Lőkös, L., 26.06.2009 [BP 93457]. - Romania, Suceava County, Călimani Mts, Călimani National Park, Mt Negoi, near the sulphur mine (Exploatarea Că- 
limani), on bark (Pinus cembra). Lat.: $47^{\circ} 06^{\prime} 24.7^{\prime \prime} \mathrm{N}$; Long.: 25 $14^{\prime} 25.3$ ' E; Alt.: 1,637 m a.s.l. Leg.: Lőkös, L., 28.06.2009 [BP 96297].

Chaenotheca furfuracea (L.) Tibell - M-ții Călimanului valea Drâglelor, 400 m. alt., pe scoarță de Fagus. Coll.: Codoreanu, V., 07.1949 [CL as Coniocybe gracilenta] (CODOREANU 1952 as C.gracilenta, Moruzi et al. 1967 as C. gracilenta, BARTó 2002 as Chaenotheca gracilenta, CiURcheA 2004 as Coniocybe gracilenta). - Romania, Suceava County, Călimani Mts, in the valley of the stream Tarnița, on bark (Picea abies). Lat.: $47^{\circ} 10^{\prime} 49.5^{\prime \prime} \mathrm{N}$; Long.: $25^{\circ} 13^{\prime} 17.6^{\prime \prime} \mathrm{E}$; Alt.: 1,121 m a.s.l. Leg.: Lőkös, L., 26.06.2009 [BP 93365].

! Chaenotheca trichialis (Ach.) Hellb. - Romania, Suceava County, Călimani Mts, in the valley of the stream Tarnița, on bark (Picea abies). Lat.: $47^{\circ} 10^{\prime} 49.5^{\prime \prime} \mathrm{N}$; Long.: 25 13' 17.6" E; Alt.: 1,121 m a.s.l. Leg.: Lőkös, L., 26.06.2009 [BP 93366]. - Romania, Suceava County, Călimani Mts, Călimani National Park, Mt Negoi, near the sulphur mine (Exploatarea Călimani), on bark (Picea abies). Lat.: $47^{\circ} 06^{\prime}$ 24.7” N; Long.: $25^{\circ} 14$ ' 25.3” E; Alt.: 1,637 m a.s.l. Leg.: Lőkös, L., 28.06.2009 [BP 96306]. - Chaenoteca trichialis f. cinerea (Pers.) Keissl. and Chaenoteca trichialis f. candelaris Schaer. were mistakenly reported by MoRUzi et al. (1967) and BARTóK (2002) from the Călimani Mts as 'Mții Călimani, lingă Tuşnad'.

Cladonia arbuscula (Wallr.) Flot. s. 1. - Distr. Mureş: Munții Călimani, Pietrosul, alt. 1,900-2,102 m s. m. Coll.: Nyárády, E. I., 22.07.1914 (CRETzoiU 1939, as C. sylvatica f. arbuscula, Cretzoiv 1940, Moruzi et al. 1967 as C. sylvatica f. arbuscula, CiUrchea 2004). - Transsilvania: M. Kelemen-hegység, Magyar Negoj, alt. 1,900-2,000 m. Leg.: Jávorka, S. and Keller, J., 15.07.1941 [BP 96313 as C. arbuscula subsp. squarrosa]. - M-ții Căliman., alt. cca 1200 m. (pe sol în jurul stânei Retițiş). Coll.: Codoreanu, V., 28.07.1949 [CL as C. sylvatica] (CodoreAnu 1952 as C. sylvatica, Moruzi et al. 1967 as C. sylvatica, CiurChea 2004). - Rezervația Bistricioru, vf. Zurzugău (STOIE 1999 as Cladina arbuscula). - Romania, Călimani, Apostoli (Gura Haitii), on soil. Lat.: $47^{\circ} 14.111^{\prime} \mathrm{N}$; Long.: $25^{\circ} 14.102$ ' E, Alt.: 1,704 m a.s.l. Leg.: Hur, J.-S. (RO-09057), 27.06.2009 [KoLRI 13015 as C. arbuscula s. 1.]. - Romania, Suceava County, Călimani Mts, near Monastery at Cerbul, growing together with Cetraria islandica on acid soil. Lat.: $47^{\circ} 13^{\prime} 57.4^{\prime \prime} \mathrm{N}$; Long.: $25^{\circ}$ 13’35.4” E; Alt.: 1,618 m a.s.l. Leg.: Lőkös, L., 27.06.2009 [BP 93483 as C. arbuscula subsp. squarrosa]. - M-ții Călimani, Parcul Național Călimani, Doisprezece Apostoli (Gura Haitii) on soil, 27.06.2009 [hb. Crişan 2253].

Cladonia bellidiflora (Ach.) Schaer. - Distr. Mureş: Munții Călimani (CRETzoIU 1940 as C. bellidiflora f. coccocephala, Moruzi et al. 1967 as C. bellidiflora f. coccocephala, Ciurchea 2004). - M-ții Călimanului, valea Drâglei, (creşte pe sol printre muşchi). Coll.: Codoreanu, V., 07.1949 [CL as C. bellidiflora f. coccocephala] (Codoreanu 1952 as C. bellidiflora f. coccocephala, Moruzi et al. 1967 as C. bellidiflora f. coccocephala, CIURCHEA 2004). 
Cladonia botrytes (K. G. Hagen) Willd. - Rezervația Pădurea din Şes, on a stump in the central swamp, 04.08.1996 [hb. Crişan 995] (STOIE and CRIŞAN 1998). - Rezervația Bistricioru, vf. Zurzugău (STOIE 1999).

Cladonia caespiticia (Pers.) Flörke - Rezervația Valea Repedea: on soil near the rock 'Deget', 21.07.1997 [hb. Crişan 1209] (STOIE and CRIŞAN 1998).

Cladonia carneola (Fr.) Fr. - Distr. Mureş: Munţii Călimani (CretzorU 1941, Moruzi et al. 1967, Ciurchea 2004). - Rezervația Bistricioru, Piatra Zurzugăului (StoIe 1999). - The record of C. carneola f. prolifera from 'Tuşnad' (CODOREANU 1954) is mistakenly reported by MorUzi et al. (1967) and CiURCHEA (2004) from the Călimani Mts.

Cladonia chlorophaea (Sommerf.) Spreng. - Rezervația Valea Repedea: on soil at the base of the rock 'Deget', 21.07.1997 [hb. Crişan 1212] (STOIE and Crişan 1998). - Romania, Călimani, Potica (Gura Haitii), on bark. Lat.: $47^{\circ}$ 11.445' N; Long.: $25^{\circ} 14.262^{\prime}$ E, Alt.: 1,100 m a.s.l. Leg.: Hur, J.-S. (RO-09040), 26.06.2009 [KoLRI 12999 as C. chlorophaea s. 1.].

! Cladonia coccifera (L.) Willd. - Romania, Călimani, Vf Rețitiş, on rock. Lat.: $47^{\circ} 05.847^{\prime} \mathrm{N}$; Long.: $25^{\circ} 14.737^{\prime}$ E, Alt.: 2,022 m a.s.l. Leg.: Hur, J.-S. (RO09026), 25.06.2009 [KoLRI 12986].

Cladonia coniocraea (Flörke) Spreng. - M-ții Călimanului, vf. Voivodesii, 1,700 m. alt. Coll.: Codoreanu, V., 07.1949 [CL] (CodoreAnU 1952, Moruzi et al. 1967, CiurChEA 2004). - Rezervaţia Valea Repedea: at the base of a spruce in 'Poiana Calului', 21.07.1997 [hb. Crişan 1230], pârâul 'Repedele', pârâul 'Scoruşet' (STOIE and CrişAN 1998). - Rezervaţia Bistricioru, Piatra Zurzugăului (STOIE 1999). - Romania, Călimani, Reservation Pinus Cembra, on bark. Lat.: $47^{\circ}$ 06.381' N; Long.: $25^{\circ} 14.411^{\prime}$ E, Alt.: 1,653 m a.s.l. Leg.: Hur, J.-S. (RO-09089), 28.06.2009 [KoLRI 13041].

Cladonia cornuta (L.) Hoffm. - M-ții Călimanului, stâna Retițiş, 1,400 m alt., (pe sol). Coll.: Codoreanu, V., 28.07.1949 [CL] (Codoreanu 1952 as C. cornuto-radiata var. subulata, MORUZI et al. 1967 as C. cornutoradiata var. subulata, Ciurchea 2004). - M-ții Căliman. Alt. cca. 1,200 m. Coll.: Codoreanu, V., 28.07.1949 [CL as C. cornuta f.phyllotoca]. - Romania, Suceava County, Călimani Mts, near Monastery at Cerbul, on acid soil. Lat.: $47^{\circ} 13^{\prime}$ 57.4” N; Long.: 25 $13^{\prime}$ 35.4” E; Alt.: 1,618 m a.s.1. Leg.: Lőkös, L., 27.06.2009 [BP 93481].

Cladonia crispata (Ach.) Flot. - Rezervația Bistricioru, vf. Zurzugău (STOIE 1999).

Cladonia deformis (L.) Hoffm. - Jud. Mureş: Munții Călimani (Cretzoiv 1940, Moruzi et al. 1967, Ciurchea 2004). - M-ții Călimanului, m-tele Puturosul la 1,100 m alt. (Codoreanu 1954 as C. deformis f. cyathiformis, Moruzi et al. 1967 as C. deformis f. cyathiformis, Ciurchea 2004). - Rezervația Valea Repedea: izvorul pârâului 'Ursului', on soil among mosses at the base of the rock 
'Deget', 21.07.1997 [hb. Crişan 1218] (STOIE and CrişAN 1998). - Rezervația Bistricioru, crestei Bistricioru-Piatra Zurzugăului (STOIE 1999).

Cladonia digitata (L.) Hoffm. - M-ții Călimanului, stâna Retițiş, 1,500 m. alt. Coll.: Codoreanu, V., 07.1949 [CL] (Codoreanu 1952, Moruzi et al. 1967, Ciurchea 2004). - M-ții Căliman., Valea Drîglelor. Alt. cca. 800 m. Coll.: Codoreanu, V., 28.07.1949 [CL]. - Rezervația Valea Repedea: at about $1 \mathrm{~km}$ downstream of the spring of stream 'Repedele', 20.07.1997 [hb. Crişan 1119], vârful 'Poiana Calului', stânca 'Cubică' (STOIE and CRIŞAN 1998). - Rezervația Bistricioru, Piatra Zurzugăului, vf. Bistricioru (STOIE 1999). - Romania, Călimani, Reservation Pinus Cembra, on bark. Lat.: $47^{\circ} 06.381^{\prime} \mathrm{N}$; Long.: $25^{\circ}$ 14.411' E, Alt.: 1,653 m a.s.l. Leg.: Hur, J.-S. (RO-09090, RO-09091), 28.06.2009 [KoLRI 13042, KoLRI 12788]. - Romania, Hargitha County, Călimani Mts, Călimani National Park, near Lake Iezer, on bark (Picea abies). Lat.: $47^{\circ} 05^{\prime} 33.6^{\prime \prime}$ N; Long.: 25 15’ 40.6” E; Alt.: 1,744 m a.s.l. Leg.: Lőkös, L., 25.06.2009 [BP 93352]. - Romania, Suceava County, Călimani Mts, near the village Gura Haitii in the valley of the stream Tarnița, on bark (Picea abies). Lat.: $47^{\circ} 11^{\prime} 22.8^{\prime \prime} \mathrm{N}$; Long.: $25^{\circ} 14$ ' 17.9" E; Alt.: 1,102 m a.s.l. Leg.: Lőkös, L., 26.06.2009 [BP 93358].

Cladonia ecmocyna Leight. - Jud. Mureş: munții Călimani pe vîrful Gruiu la ca 1,700 m. pe sol intre Cetraria islandica, în massive de Pinus mughus (CretzorU and Klement 1935, Moruzi et al. 1967, Ciurchea 2004).

Cladonia fimbriata (L.) Fr. - M-ții Călimanului. Coll.: Codoreanu, V., 07.1949 [CL]. - M-ții Căliman., Pietrosul. Alt. cca. 1,900 m. Coll.: Codoreanu, V., 27.07.1949 [CL]. - M-ții Căliman. Coll.: Codoreanu, V., 28.07.1950 [CL] (Codoreanu 1952, Moruzi et al. 1967 as C. fimbriata var. simplex, Ciurchea 2004). - Rezervația Pădurea din Şes \& Rezervația Valea Repedea, on the bark at the base of tree trunks and on soil, 04.08.1996 [hb. Crişan 790] (STOIE and CRIŞAN 1998). - Rezervația Bistricioru, vf. Zurzugău spre Poiană (STOIE 1999). - Romania, Călimani, Vf Negoi Romanesc, on soil. Lat.: $47^{\circ} 07.286^{\prime}$ N; Long.: $25^{\circ} 13.348^{\prime}$ E, Alt.: 1,680 m a.s.l. Leg.: Hur, J.-S. (RO-09002), 25.06.2009 [KoLRI 12970].

Cladonia floerkeana (Fr.) Flörke - Rezervația Bistricioru, vf. Zurzugău (STOIE 1999).

Cladonia furcata (Huds.) Schrad. - Rezervația Valea Repedea: stânca 'Deget', pârâul 'Scoruşet' (STOIE and CRIŞAN 1998). - Rezervația Bistricioru, pe vârfurile Zurzugău şi Bistricioru (STOIE 1999). - Romania, Călimani, Apostoli (Gura Haitii), on soil. Lat.: $47^{\circ} 13.964^{\prime} \mathrm{N}$; Long.: $25^{\circ} 13.612^{\prime} \mathrm{E}$, Alt.: $1,752 \mathrm{~m}$ a.s.l. Leg.: Hur, J.-S. (RO-09059), 27.06.2009 [KoLRI 13017]. - Romania, Călimani, Tarnița (Gura Haitii), on soil. Lat.: $47^{\circ} 08.980^{\prime}$ N; Long.: $25^{\circ} 11.640^{\prime}$ E, Alt.: 1,377 m a.s.l. Leg.: Hur, J.-S. (RO-09048), 26.06.2009 [KoLRI 13006]. Romania, Suceava County, Călimani Mts, in the valley of the stream Tarnița, on mossy soil. Lat.: $47^{\circ} 10^{\prime} 37.1^{\prime \prime} \mathrm{N}$; Long.: $25^{\circ} 13^{\prime} 08.3^{\prime \prime} \mathrm{E}$; Alt.: $1,345 \mathrm{~m}$ a.s.l. Leg.: 
Lőkös, L., 26.06.2009 [BP 93464]. - Romania, Suceava County, Călimani Mts, Călimani National Park, along tourist path between Stâncile Lucaciu and Stâncile Doisprezece Apostoli, on acid soil. Lat.: $47^{\circ} 13^{\prime} 27.9^{\prime \prime} \mathrm{N}$; Long.: $25^{\circ} 12^{\prime} 41.5^{\prime \prime} \mathrm{E}$; Alt.: 1,726 m a.s.l. Leg.: Lőkös, L., 27.06.2009 [BP 93490]. - M-ții Călimani Mts, Parcul Naţional Călimani, at Stâncile Doisprezece Apostoli, on soil, 27.06.2009 [hb. Crişan 2256].

Cladonia glauca Flörke - M-ții Călimanului, vf. Pietrosul, 1,500 m. alt., (pe sol între muşchi). Coll.: Codoreanu, V., 07.1949 [CL] (Codoreanu 1952, MoRUZI et al. 1967, CIURCHEA 2004).

Cladonia gracilis (L.) Willd. - M-ții Călimanului, vf. Bradul Ciont, 1,800 m alt. Coll.: Codoreanu, V., 29.07.1949 [CL] (Codoreanu 1952 as C. elongata var. esquamosa f. exoncera, Moruzi et al. 1967 as C. gracilis var. esquamosa f. exoncera, Ciurchea 2004). - Rezervația Bistricioru, vf. Zurzugău (Stoie 1999). - Romania, Călimani, Apostoli (Gura Haitii), on moss. Lat.: $47^{\circ} 13.944$ N ; Long.: $25^{\circ} 13.466^{\prime}$ E, Alt.: 1,732 m a.s.l. Leg.: Hur, J.-S. (RO-09069), 27.06.2009 [KoLRI 12783]. - Romania, Călimani, Vf Rețitiş, on soil. Lat.: $47^{\circ} 05.847^{\prime} \mathrm{N}$; Long.: $25^{\circ} 14.737^{\prime}$ E, Alt.: 2,022 m a.s.l. Leg.: Hur, J.-S. (RO-09029), 25.06.2009 [KoLRI 12989]. - Romania, Călimani, Vf Rețitiş, on moss. Lat.: $47^{\circ} 05.689^{\prime} \mathrm{N}$; Long.: $25^{\circ}$ 15.758' E, Alt.: 1,737 m a.s.l. Leg.: Hur, J.-S. (RO-09032, RO-09033), 25.06.2009 [KoLRI 12992, KoLRI 12993]. - Romania, Suceava County, Călimani Mts, Călimani National Park, Mt Rețitiş, near the meteorological station (Stația Meteo), ca $11 \mathrm{~km} \mathrm{SSW}$ of Gura Haitii, on acid soil. Lat.: $47^{\circ} 05^{\prime} 51.1^{\prime \prime} \mathrm{N}$; Long.: $25^{\circ} 14$ ' 46.1" E; Alt.: 2,006 m a.s.l. Leg.: Lőkös, L., 25.06.2009 [BP 93335].

Cladonia macilenta Hoffm. - Rezervația Valea Repedea: among mosses near the rock 'Deget', 10.08.1996 [hb. Crişan 819] (STOIE and CRişAN 1998).

Cladonia parasitica (Hoffm.) Hoffm. - Rezervația Valea Repedea: pârâul 'Repedele', on a stump above the rock 'Deget', 21.07.1997 [hb. Crişan 1215] (STOIE and CrişAN 1998). - Rezervaţia Bistricioru, vf. Zurzugău (STOIE 1999).

Cladonia pleurota (Flörke) Schaer. - Jud. Mureş: munții Călimani pe vîrful Gruiu, pe sol, la ca 1,700 m. alt. (CretzoiU and Klement 1935, CretzoiU 1941, Moruzi et al. 1967, CiUrchea 2004). - Rezervația Valea Repedea: among mosses at the base of the rock 'Cubică', 10.08 .1996 [hb. Crişan 811] (STOIE and CrişAN 1998). - Rezervaţia Bistricioru (Stole 1999). - Romania, Călimani, Apostoli (Gura Haitii), on soil. Lat.: $47^{\circ} 14.473^{\prime}$ N; Long.: $25^{\circ} 14.455^{\prime}$ E, Alt.: 1,664 m a.s.l. Leg.: Hur, J.-S. (RO-09054), 27.06.2009 [KoLRI 13012]. - Romania, Călimani, Vf Negoi Romanesc, on soil. Lat.: $47^{\circ} 07.286^{\prime} \mathrm{N}$; Long.: $25^{\circ} 13.348^{\prime} \mathrm{E}$, Alt.: 1,680 m a.s.l. Leg.: Hur, J.-S. (RO-09011), 25.06.2009 [KoLRI 12776].

Cladonia polydactyla (Flörke) Spreng. - Rezervația Valea Repedea: on a stump at $100 \mathrm{~m}$ above the rock 'Deget', 21.07.1997 [hb. Crişan 1216] (STOIE and CrişAN 1998). - Rezervaţia Bistricioru, Piatra Zurzugăului (Stoie 1999). 
Cladonia pyxidata (L.) Hoffm. - Rezervația Valea Repedea (Store and CrişAN 1998). - Rezervația Bistricioru (STOIE 1999). - Romania, Călimani, Apostoli (Gura Haitii), on moss. Lat.: 47 $13.944^{\prime}$ N; Long.: 25 $13.466^{\prime}$ E, Alt.: 1,732 m a.s.l. Leg.: Hur, J.-S. (RO-09067), 27.06.2009 [KoLRI 13025]. - Romania, Călimani, Reservation Pinus Cembra, on moss. Lat.: $47^{\circ} 06.381^{\prime} \mathrm{N}$; Long.: $25^{\circ}$ 14.411' E, Alt.: 1,653 m a.s.l. Leg.: Hur, J.-S. (RO-09086), 28.06.2009 [KoLRI 13038]. - Romania, Călimani, Vf Negoi Romanesc, on soil. Lat.: $47^{\circ} 07.286^{\prime} \mathrm{N}$; Long.: $25^{\circ} 13.348^{\prime}$ E, Alt.: 1,680 m a.s.l. Leg.: Hur, J.-S. (RO-09003), 25.06.2009 [KoLRI 12971]. - M-ții Călimani, Parcul Național Călimani, Gura Haitii, on moss, 27.06.2009 [hb. Crişan 2257].

Cladonia ramulosa (With.) J. R. Laundon - Rezervația Bistricioru, vf. Zurzugău, vf. Bistricioru (STOIE 1999).

Cladonia rangiferina (L.) F. H. Wigg. - Distr. Mureş: munții Călimani, Pietrosul, alt. 1,900-2,102 m s. m. Coll.: Nyárády, E. I., 22.07.1914 (CRETzorU 1939 as C. rangiferina f. cymosa, MoRUzi et al. 1967 as C. rangiferina f. cymosa, Ciurchea 2004). - M-ții Călimanului, Vf. Voivodesii, 1,800 m alt. (Codoreanu 1952, Moruzi et al. 1967, Ciurchea 2004). - Rezervația Bistricioru, vf. Zurzugău (Store 1999 as Cladina rangiferina). - Romania, Călimani, Apostoli (Gura Haitii), on soil. Lat.: $47^{\circ} 14.177^{\prime} \mathrm{N}$; Long.: $25^{\circ} 14.183^{\prime} \mathrm{E}$, Alt.: 1,692 m a.s.l. Leg.: Hur,J.-S. (RO-09056), 27.06.2009 [KoLRI 13014]. - Romania, Călimani, Apostoli (Gura Haitii), on soil. Lat.: $47^{\circ} 13.982$ ' N; Long.: $25^{\circ} 13.774$ ' E, Alt.: 1,722 m a.s.l. Leg.: Hur, J.-S. (RO-09058), 27.06.2009 [KoLRI 13016]. - Romania, Călimani, Vf Rețitiş, on moss. Lat.: $47^{\circ} 05.689^{\prime} \mathrm{N}$; Long.: $25^{\circ} 15.758^{\prime} \mathrm{E}$, Alt.: $1,737 \mathrm{~m}$ a.s.l. Leg.: Hur, J.-S. (RO-09034), 25.06.2009 [KoLRI 12994]. - Romania, Călimani, Vf Rețitiş, on soil. Lat.: $47^{\circ} 05.847^{\prime}$ N; Long.: $25^{\circ} 14.737^{\prime} \mathrm{E}$, Alt.: 2,022 m a.s.l. Leg.: Hur, J.-S. (RO-09022), 25.06.2009 [KoLRI 12982]. - Romania, Suceava County, Călimani Mts, near Monastery at Cerbul, growing together with Cetraria islandica on acid soil. Lat.: $47^{\circ} 13^{\prime}$ 57.4" N; Long.: $25^{\circ} 13^{\prime} 35.4$ " E; Alt.: 1,618 m a.s.l. Leg.: Lőkös, L., 27.06.2009 [BP 93482].

Cladonia squamosa (Scop.) Hoffm. - M-ții Călimanului, Vf. Pietrosul, pe sol pietros (CODOREANu 1952 as C. subsquamosa f. luxurians, MORUzi et al. 1967 as C. subsquamosa f. luxurians, CiURCHEA 2004). Rezervația Valea Repedea: pârâul 'Scoruşet' (STOIE and CRIŞAN 1998).

Cladonia stygia (Fr.) Ruoss - Rezervația Bistricioru, vf. Zurzugău, Piatra Zurzugăului (STOIE 1999).

Cladonia subulata (L.) F. H. Wigg. - M-ții Călimanului, stâna Retițiş, 1,400 m alt., (pe sol). Coll.: Codoreanu, V., 28.07.1949 [CL as C. cornuto-radiata var. subulata] (CODOREANU 1952 as C. cornuto-radiata var. subulata, Moruzi et al. 1967 as C. cornutoradiata var. subulata, Ciurchea 2004). - M-ții Călimanului, vf. Bradul Ciont, 1,900 m alt. Coll.: Codoreanu, V., 28.07.1949 [CL as C. cornuto- 
radiata var. radiata] (CODOREANU 1952 as C. cornuto-radiata var. radiata, MoRUZI et al. 1967 as C. cornutoradiata f. radiata, CiurChea 2004). - Rezervația Valea Repedea: izvorul pârâului 'Repedele', on soil among mosses at the base of the rock 'Deget', 21.07.1997 [hb. Crişan 1224] (STOIE and CRişAN 1998).

Cladonia sulphurina (Michx.) Fr. - Rezervația Bistricioru, vf. Zurzugău, Piatra Zurzugăului (Stoie 1999). - Romania, Călimani, Vf Rețitiş, on moss. Lat.: $47^{\circ} 05.847^{\prime} \mathrm{N}$; Long.: $25^{\circ} 14.737^{\prime} \mathrm{E}$, Alt.: 2,022 m a.s.l. Leg.: Hur, J.-S. (RO09031), 25.06.2009 [KoLRI 12991].

!!* Clypeococcum hypocenomycis D. Hawksw. - Romania, Suceava County, Călimani Mts, Călimani National Park, Mt Negoi, near the sulphur mine (Exploatarea Călimani), on bark (Pinus cembra), growing on Hypocenomyce scalaris. Lat.: $47^{\circ} 06^{\prime} 24.7^{\prime \prime} \mathrm{N}$; Long.: $25^{\circ} 14^{\prime} 25.3$ ” E; Alt.: 1,637 m a.s.l. Leg.: Lőkös, L., 28.06.2009 [BP 96298].

! Coenogonium pineti (Ach.) Lücking et Lumbsch - Romania, Suceava County, Călimani Mts, in the valley of the stream Tarnița, on bark (Picea abies). Lat.: $47^{\circ} 10^{\prime} 49.5^{\prime \prime} \mathrm{N}$; Long.: $25^{\circ} 13^{\prime} 17.6^{\prime \prime} \mathrm{E}$; Alt.: 1,121 m a.s.l. Leg.: Lőkös, L., 26.06.2009 [BP 93367].

Collema flaccidum (Ach.) Ach. - M-ții Călimanului, Drâglele, $650 \mathrm{~m}$ alt. Coll.: Codoreanu, V., 07.1949 [CL 217886 as C. rupestre] (CodoreAnu 1952 as C. rupestre, Moruzi et al. 1967 as C. rupestre, BARTó K 1999, CiURCHEA 2004).

Collema nigrescens (Huds.) DC. - M-ții Călimanului, valea Drâglelor, 600 m. alt., pe scoarță de Fagus silvatica. Coll.: Codoreanu, V., 07.1949 [CL] (CodoREANU 1952, Moruzi et al. 1967, BARTó K 1999, CiURCheA 2004).

Cornicularia normoerica (Gunnerus) Du Rietz - M-ții Călimanului, vf. Voivodesii, 1,885 m. alt. Coll.: Codoreanu, V., 07.1949 [CL as Cetraria tristis] (Codoreanu 1952 as Cetraria tristis, Moruzi et al. 1967, Ciurchea 2004).

! Dibaeis rosea (Pers.) Clem. - Romania, Călimani, Apostoli (Gura Haitii), on soil. Lat.: $47^{\circ} 13.941^{\prime} \mathrm{N}$; Long.: $25^{\circ} 13.552^{\prime} \mathrm{E}$, Alt.: $1,733 \mathrm{~m}$ a.s.l. Leg.: Hur, J.-S. (RO-09063), 27.06.2009 [KoLRI 13021 as Dibaeis baeomyces]. - Romania, Suceava County, Călimani Mts, Mt Negoi, near the sulphur mine (Exploatarea Călimani), ca 9 km SSW of Gura Haitii, on acid soil. Lat.: $47^{\circ} 07^{\prime} 17.3^{\prime \prime}$ N; Long.: $25^{\circ} 13^{\prime}$ 20.0” E; Alt.: 1,673 m a.s.l. Leg.: Lőkös, L., 25.06.2009 [BP 93329]. Romania, Suceava County, Călimani Mts, Călimani National Park, Mt Rețitiş, near the meteorological station (Staţia Meteo), ca $11 \mathrm{~km} \mathrm{SSW}$ of Gura Haitii, on acid soil. Lat.: $47^{\circ} 05^{\prime}$ 51.1" N; Long.: $25^{\circ} 14^{\prime} 46.1$ " E; Alt.: 2,006 m a.s.l. Leg.: Lőkös, L., 25.06.2009 [BP 93333]. - M-ții Călimani, Parcul Naţional Călimani, Vf. Rețitiş, near the meteorological station, on soil, 25.06.2009 [hb. Crişan 2204]. - Romania, Suceava County, Călimani Mts, Călimani National Park, along tourist path between Stâncile Lucaciu and Stâncile Doisprezece Apostoli, on acid soil. 
Lat.: $47^{\circ} 13^{\prime} 27.9^{\prime \prime} \mathrm{N}$; Long.: $25^{\circ} 12^{\prime}$ 41.5” E; Alt.: 1,726 m a.s.l. Leg.: Lökös, L., 27.06.2009 [BP 93491].

Diploschistes muscorum (Scop.) R. Sant. - M-ții Călimanului, valea Drâglelor, 450 m alt., pe muşchi. Coll.: Codoreanu, V., 07.1949 [CL as D. bryophilus] (CODOREANu 1952 as D. bryophilus, Moruzi et al. 1967 as D. bryophilus, CiURCHEA 2004).

Enchylium polycarpon (Hoffm.) Otálora, P. M. Jørg. et Wedin - M-ții Călimanului, vf. Pietrosul, 2,100 m. alt. Coll.: Codoreanu, V., 07.1949 [CL 217874 as Collema polycarpum] (CODOREANU 1952 as C. polycarpum, MORUZI et al. 1967 as C. polycarpum, BARTó K 1999 as C. polycarpon, CiURCHEA 2004 as C.polycarpon).

!!* Endococcus macrosporus (Arnold) Nyl. - Romania, Suceava County, Călimani Mts, Călimani National Park, Mt Rețitiş, near the meteorological station (Stația Meteo), ca $11 \mathrm{~km} \mathrm{SSW}$ of Gura Haitii, on siliceous rock. Lat.: $47^{\circ} 05^{\prime}$ 51.1” N; Long.: 25 14’ 46.1” E; Alt.: 2,006 m a.s.l. Leg.: Lőkös, L., 25.06.2009 [BP 93346] (Fig. 2) (cf. SÉRUSIAUX et al. 1999).

Evernia divaricata (L.) Ach. - M-ții Călimanului. Coll.: Codoreanu, V., 07.1949 [CL as Letharia divaricata] (CODOREANU 1952 as L. divaricata, MORU-

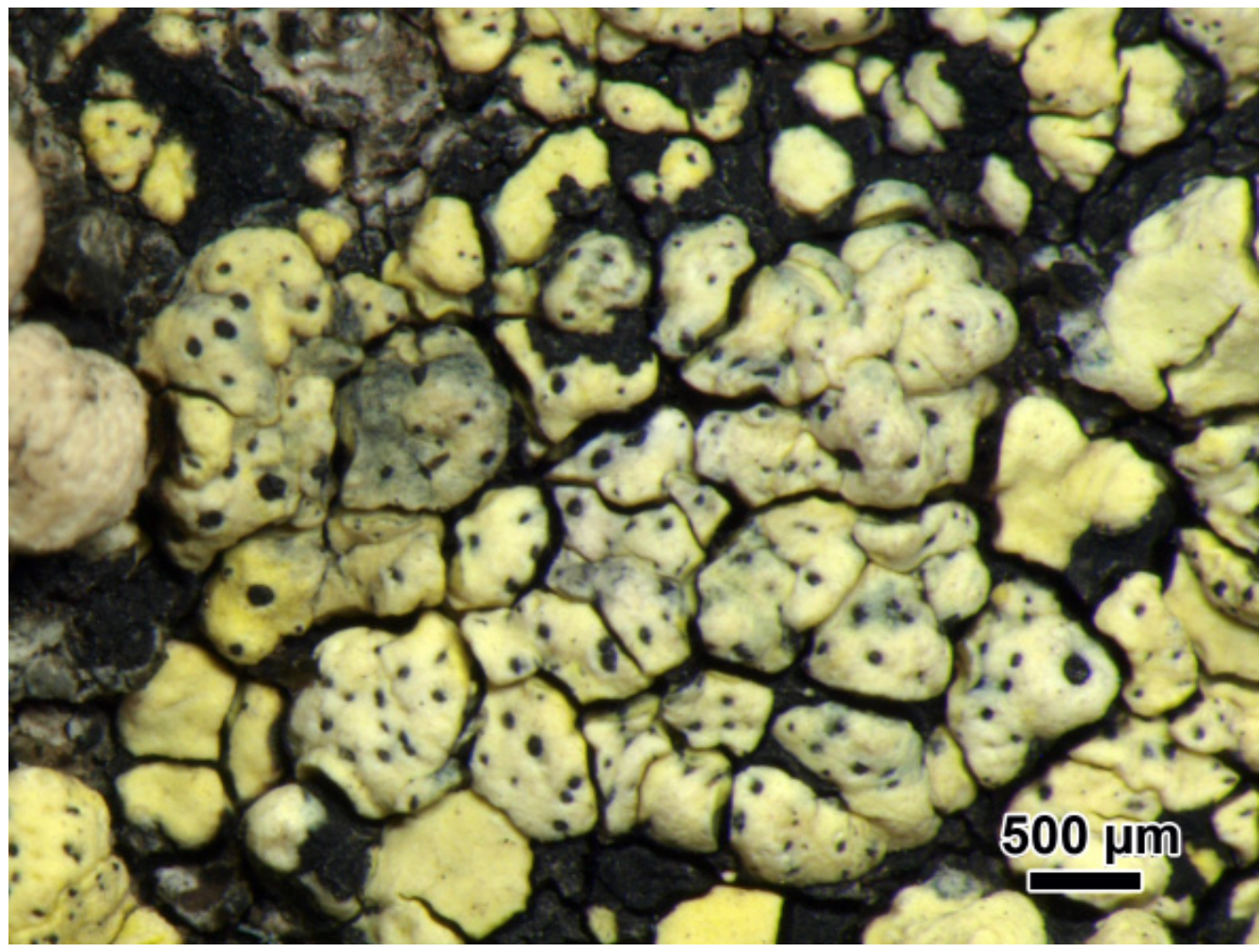

Fig. 2. Black, immersed perithecioid pseudothecia of Endococcus macrosporus, a lichenicolous microfungi, growing on Rhizocarpon geographicum from the recent collection (BP 93346). 
Zi et al. 1967, Ciurchea 2004). - M-ții Căliman., Drâglele, (pe ramurile coniferelor). Coll.: Codoreanu, V., 28.07.1949 [CL 631121 as L. divaricata] (Fig. 3) (Codoreanu 1952 as L. divaricata, Moruzi et al. 1967, Ciurchea 2004). Rezervația Bistricioru (STOIE 1999). - Romania, Călimani, Tarnița (Gura Haitii), on twig. Lat.: $47^{\circ} 10.652^{\prime} \mathrm{N}$; Long.: $25^{\circ} 13.139^{\prime} \mathrm{E}$, Alt.: $1,342 \mathrm{~m}$ a.s.l. Leg.: Hur, J.-S. (RO-09052), 26.06.2009 [KoLRI 13010]. - Romania, Călimani, Vf Negoi Romanesc, on bark. Lat.: $47^{\circ} 07.286^{\prime} \mathrm{N}$; Long.: $25^{\circ} 13.348^{\prime}$ E, Alt.: 1,680 m a.s.l. Leg.: Hur, J.-S. (RO-09008), 25.06.2009 [KoLRI 12974]. - Romania, Hargitha County, Călimani Mts, Călimani National Park, near Lake Iezer, on bark (Picea abies). Lat.: $47^{\circ} 05^{\prime} 33.6^{\prime \prime} \mathrm{N}$; Long.: $25^{\circ} 15^{\prime} 40.6^{\prime \prime} \mathrm{E}$; Alt.: 1,744 m a.s.l. Leg.: Lőkös, L., 25.06.2009 [BP 93355]. - M-ții Călimani, Parcul Național Călimani, near Lake Iezer, on bark of spruce, 25.06.2009 [hb. Crişan 2205]. - Romania, Suceava County, Călimani Mts, Călimani National Park, along tourist path between Stâncile Lucaciu and Stâncile Doisprezece Apostoli, on siliceous rock. Lat.: $47^{\circ}$ 13’27.9” N; Long.: 25 12' 41.5” E; Alt.: 1,726 m a.s.l. Leg.: Lőkös, L., 27.06.2009 [BP 93498].

Evernia prunastri (L.) Ach. - Distr. Bistrița-Năsăud: Munții Călimani, pe Fagus. Leg.: M. Stoian. (Cretzoiu 1931, Moruzi et al. 1967, Ciurchea 2004). - Distr. Bistrița-Năsăud: munții Călimani, pe Fagus. Leg.: M. Stoian. (CRETzorU 1931 as E. prunastri f. sorediifera, MoRUZI et al. 1967 as E. prunastri f. sorediifera, Ciurche a 2004). - Valea Drâglele, 400 m alt. (Codoreanu 1952, Moruzi $e t$ al. 1967, Ciurchea 2004). - Rezervația Pădurea din Şes, on oak bark, 04.08.1996 [hb. Crişan 783] (STOIE and CRIŞAN 1998).

Flavoparmelia caperata (L.) Hale - Jud. Mureş: Munții Călimani (CodoreANU 1952 as Parmelia caperata, Moruzi et al. 1967 as P. caperata, CiUnChe A 2004). - Rezervația Pădurea din Şes, on oak bark, 04.08 .1996 [hb. Crişan 788] (Stoie and Crişan 1998).
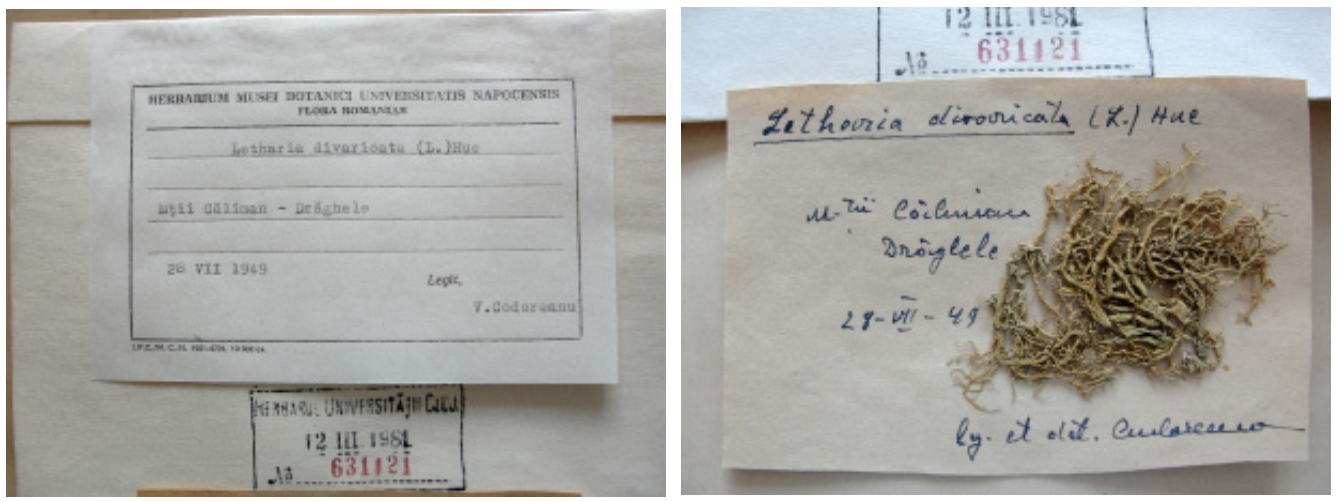

Fig. 3. Letharia divaricata (= Evernia divaricata) specimen collected by V. Codoreanu in 1949 (CL 631121). 
! Hypocenomyce scalaris (Lilj.) M. Choisy - Romania, Suceava County, Călimani Mts, Călimani National Park, Mt Negoi, near the sulphur mine (Exploatarea Călimani), on bark (Pinus cembra). Lat.: $47^{\circ} 06^{\prime} 24.7^{\prime \prime}$ N; Long.: $25^{\circ} 14^{\prime} 25.3^{\prime \prime}$ E; Alt.: 1,637 m a.s.l. Leg.: Lőkös, L., 28.06.2009 [BP 96298].

Hypogymnia farinacea Zopf - Rezervația Bistricioru (Stole 1999). - Romania, Călimani, Reservation Pinus Cembra, on bark. Lat.: $47^{\circ} 06.381$ ' N; Long.: $25^{\circ}$ 14.411' E, Alt.: 1,653 m a.s.l. Leg.: Hur, J.-S. (RO-09092), 28.06.2009 [KoLRI 13043]. - Romania, Suceava County, Călimani Mts, Călimani National Park, Mt Negoi, near the sulphur mine (Exploatarea Călimani), on bark (Picea abies). Lat.: $47^{\circ} 06^{\prime} 24.7^{\prime \prime} \mathrm{N}$; Long.: $25^{\circ} 14^{\prime} 25.3$ " E; Alt.: 1,637 m a.s.l. Leg.: Lőkös, L., 28.06.2009 [BP 96307].

Hypogymnia physodes (L.) Nyl. - Rezervaţia Pădurea din Şes \& Rezervația Valea Repedea (Stoie and Crişan 1998). - Rezervația Bistricioru (Stoie 1999). - Romania, Călimani, Apostoli (Gura Haitii), on rock. Lat.: $47^{\circ} 13.453^{\prime}$ N; Long.: $25^{\circ} 12.715^{\prime}$ E, Alt.: 1,740 m a.s.l. Leg.: Hur, J.-S. (RO-09079), 27.06.2009 [KoLRI 13032 ]. - Romania, Călimani, Tarnița (Gura Haitii), on twig. Lat.: $47^{\circ} 10.652^{\prime}$ N; Long.: $25^{\circ} 13.139$ ' E, Alt.: 1,342 m a.s.l. Leg.: Hur, J.-S. (RO-09051), 26.06.2009 [KoLRI 13009]. - Romania, Călimani, Vf Negoi Romanesc, on bark. Lat.: $47^{\circ}$ 07.286' N; Long.: $25^{\circ} 13.348^{\prime}$ E, Alt.: 1,680 m a.s.l. Leg.: Hur, J.-S. (RO-09009), 25.06.2009 [KoLRI 12975]. - Romania, Călimani, Vf Rețitiş, on twig. Lat.: $47^{\circ}$ 05.847’ N; Long.: $25^{\circ} 14.737^{\prime}$ E, Alt.: 2,022 m a.s.l. Leg.: Hur, J.-S. (RO-09027), 25.06.2009 [KoLRI 12987]. - M-ții Călimani, Parcul Național Călimani, Vf. Rețitiş, on twigs, 25.06.2009 [hb. Crişan 2211]. - Romania, Suceava County, Călimani Mts, in the valley of the stream Tarnița, on bark (Salix). Lat.: $47^{\circ} 10^{\prime}$ 37.1” N; Long.: 25 13' 08.3” E; Alt.: 1,345 m a.s.l. Leg.: Lőkös, L., 26.06.2009 [BP 93466]. - Romania, Suceava County, Călimani Mts, in the village Gura Haitii. Riverside trees along the river Neagra Şarului. On bark (Alnus). Lat.: $47^{\circ} 14$ ' 35.0” N; Long.: $25^{\circ} 14^{\prime} 28.8^{\prime \prime}$ E; Alt.: 1,026 m a.s.l. Leg.: Lőkös, L., 27.06.2009 [BP 93472]. - Romania, Suceava County, Călimani Mts, Călimani National Park, along tourist path between Stâncile Lucaciu and Stâncile Doisprezece Apostoli, on siliceous rock. Lat.: $47^{\circ} 13^{\prime} 27.9^{\prime \prime} \mathrm{N}$; Long.: $25^{\circ} 12^{\prime} 41.5^{\prime \prime} \mathrm{E}$; Alt.: $1,726 \mathrm{~m}$ a.s.l. Leg.: Lőkös, L., 27.06.2009 [BP 96282].

Hypogymnia tubulosa (Schaer.) Hav. - Rezervația Valea Repedea: pârâul 'Repedele' (STOIE and CrişAn 1998). - Rezervația Bistricioru, pârâului Colbu (Store 1999). - Romania, Suceava County, Călimani Mts, in the village Gura Haitii. Riverside trees along the river Neagra Şarului. On bark (Alnus). Lat.: $47^{\circ}$ 14' 35.0” N; Long.: 25 14’ 28.8” E; Alt.: 1,026 m a.s.l. Leg.: Lőkös, L., 27.06.2009 [BP 93473]. - M-ții Călimani, Parcul Național Călimani, near Monastery 'Apa Rece', on bark 27.06.2009 [hb. Crişan 2259]. 
! Hypogymnia vittata (Ach.) Parrique - Romania, Suceava County, Călimani Mts, Călimani National Park, along tourist path between Stâncile Lucaciu and Stâncile Doisprezece Apostoli, on siliceous rock. Lat.: $47^{\circ} 13^{\prime} 27.9^{\prime \prime} \mathrm{N}$; Long.: $25^{\circ}$ 12' 41.5” E; Alt.: 1715 m a.s.l. Leg.: Lőkös, L., 27.06.2009 [BP 96283].

Icmadophila ericetorum (L.) Zahlbr. - Distr. Mureş: Călimani-Gebirge, im oberem Teile des Tihu-Tales, im Fichtenwald auf Boden (SERVít and CretzoiU 1937 as I. ericetorum var. viridula, MoRUZI et al. 1967 as I. ericetorum var. viridula, Ciurchea 2004). - M-ții Călimanului, Drâglele, 800 m. alt. Coll.: Codoreanu, V., 07.1949 [CL]. - M-ții Călimanului, Vf. Bradul Ciont, 1,800 m alt. (CoDoreanu 1952, Moruzi et al. 1967, Ciurchea 2004). - Romania, Suceava County, Călimani Mts, Călimani National Park, Mt Negoi, near the sulphur mine (Exploatarea Călimani), on decaying wood. Lat.: $47^{\circ} 06^{\prime} 24.7^{\prime \prime} \mathrm{N}$; Long.: $25^{\circ} 14^{\prime}$ 25.3" E; Alt.: 1,637 m a.s.l. Leg.: Lőkös, L., 28.06.2009 [BP 96302].

Lathagrium cristatum (L.) Otálora, P. M.Jørg. et Wedin - M-ții Călimanului, stâna Retițiş, (circa 1,400 m alt.). Coll.: Codoreanu, V., 07.1949 [CL 217886 as Collema multifidum var. marginale] (CODOREANU 1952 as C. multifidum var. marginale, MORUZI et al. 1967 as C. multifidum var. marginale, BARTÓK 1999 as C. cristatum, Ciurchea 2004 as C. cristatum).

Lecanora intricata (Ach.) Ach. - Distr. Mureş: munții Călimani pe vârful Pietrosul, 2,100 m. s. m., pe roce eruptive (CRETzoiv 1943a, Moruzi et al. 1967, Ciurchea 2004).

Lecanora intumescens (Rebent.) Rabenh. - M-ții Călimanului, vf. Pietrosul, 1,700 m. alt. Coll.: Codoreanu, V., 07.1949 [CL] (CodoreAnu 1952, Moruzi et al. 1967, CIURCHEA 2004).

! Lecanora lojkaeana Szatala - Romania, Suceava County, Călimani Mts, Călimani National Park, along tourist path between Stâncile Lucaciu and Stâncile Doisprezece Apostoli, on siliceous rock. Lat.: 47 13'27.9” N; Long.: $25^{\circ} 12^{\prime} 41.5^{\prime \prime}$ E; Alt.: 1,726 m a.s.l. Leg.: Lőkös, L., 27.06.2009 [BP 96285].

Lecanora polytropa (Ehrh.) Rabenh. - Distr. Mureş: munţii Călimani pe vârful Pietrosul, pe roce eruptive, 2,100 m. s. m. (Cretzoiv 1943a, Moruzi et al. 1967, Ciurchea 2004). - Romania, Suceava County, Călimani Mts, Călimani National Park, along tourist path between Stâncile Lucaciu and Stâncile Doisprezece Apostoli, on siliceous rock. Lat.: $47^{\circ} 13^{\prime} 27.9^{\prime \prime} \mathrm{N}$; Long.: $25^{\circ} 12^{\prime} 41.5^{\prime \prime} \mathrm{E}$; Alt.: 1,726 m a.s.1. Leg.: Lőkös, L., 27.06.2009 [BP 96286].

Lecidea confluens (Weber) Ach. - M-ții Călimanului, vf. Pietrosul, 1,900 m. alt., (creşte pe pietre silicoase). Coll.: Codoreanu, V., 07.1949 [CL 217879 as L. confluens f. plana] (Codoreanu 1952 as L. confluens f. plana, Moruzi et al. 1967 as L. confluens f. plana, CiUrchea 2004). - Romania, Călimani, Vf Negoi Romanesc, on rock. Lat.: $47^{\circ} 07.286^{\prime}$ N; Long.: $25^{\circ} 13.348^{\prime}$ E, Alt.: 1,680 m a.s.l. Leg.: Hur, J.-S. (RO-09004), 25.06.2009 [KoLRI 12774]. - Romania, Călimani, 
Vf Rețitiş, on rock. Lat.: $47^{\circ} 05.847^{\prime} \mathrm{N}$; Long.: $25^{\circ} 14.737^{\prime} \mathrm{E}$, Alt.: $2,022 \mathrm{~m}$ a.s.l. Leg.: Hur, J.-S. (RO-09016), 25.06.2009 [KoLRI 12778].

Lecidea ochrocarpa (Körb.) Lettau - Jud. Mureş: Munții Călimani, m-tele Puturosul la 1,200 m alt. (Codoreanu 1954, Moruzi et al. 1967, Ciurchea 2004).

Lecidella elaeochroma (Ach.) M. Choisy - M-ții Călimanului, valea Drâglelor (CODOREANU 1952 as Lecidea parasema f. euphorea, MORUzi et al. 1967 as L. granulosa, CIURCHEA 2004 as Trapeliopsis granulosa).

Lepraria lobificans Nyl. - M-ții Călimanului, valea Drâglelor, 700 m. alt. Coll.: Codoreanu, V., 10.07.1949 [CL as Crocynia aeruginosa] (CodoreANU 1952 as C. aeruginosa, Moruzi et al. 1967 as C. aeruginosa, Ciurchea 2004 as $L$. finkii). - Romania, Suceava County, Călimani Mts, northern side of Vârful Haitii in the valley of the stream Tarnița, on bark (Picea abies) and also on acid soil. Lat.: $47^{\circ} 09^{\prime} 00.3$ ” N; Long.: $25^{\circ} 11^{\prime}$ 41.4" E; Alt.: 1,341 m a.s.l. Leg.: Lőkös, L., 26.06.2009 [BP 93460].

Lobaria pulmonaria (L.) Hoffm. - M-ții Călimanului, valea Drâglelor, 700 m. alt., (pe scoarță de Fagus, Acer şi chiar Abies). Coll.: Codoreanu, V., 30.07.1949 [CL] (Fig. 4) (CODOREANu 1952, Moruzi et al. 1967, BARTó K 1994, CiURCHEA 2004).

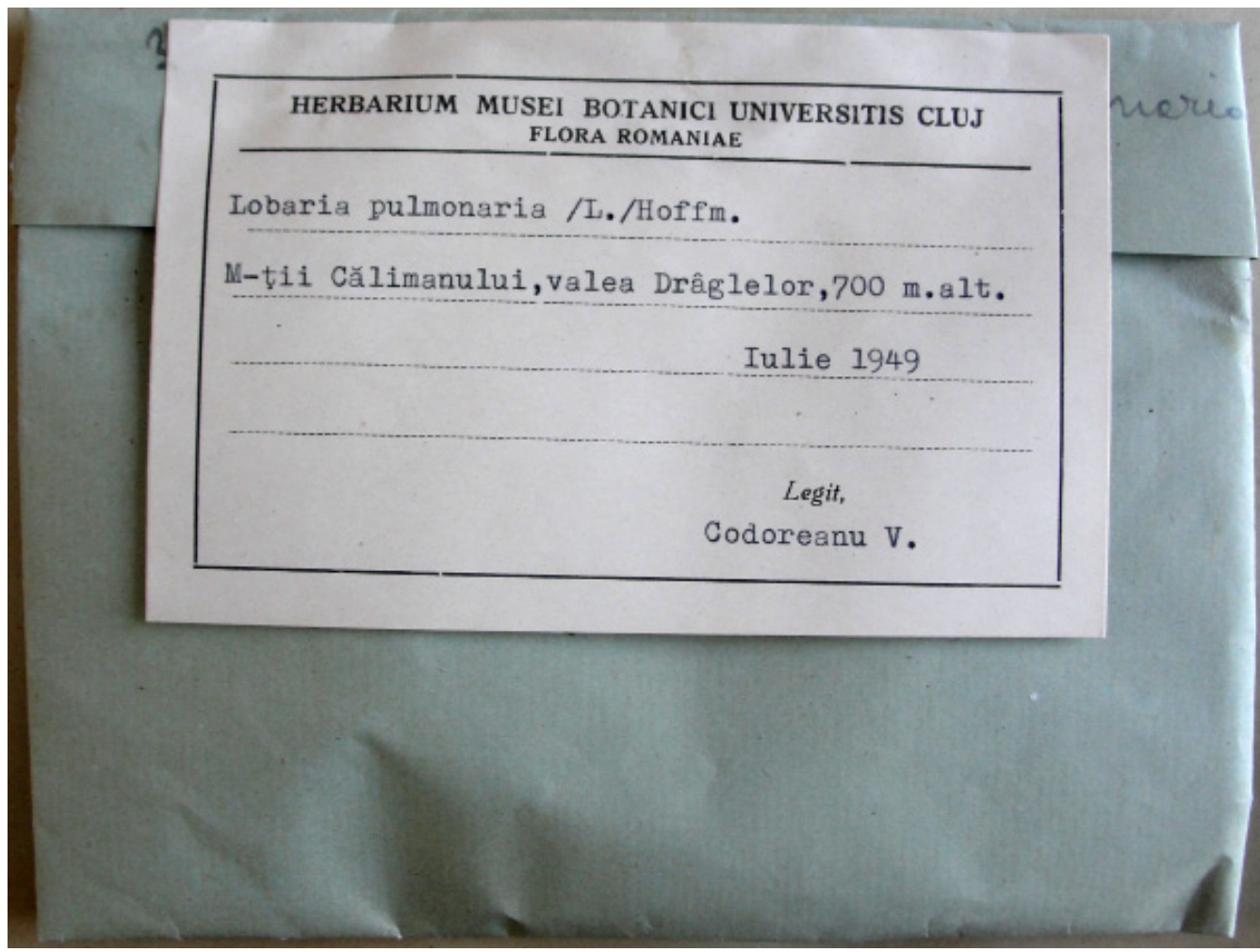

Fig. 4. Label of the Lobaria pulmonaria specimen collected by V. Codoreanu in 1949 (CL). 
! Massjukiella candelaria (L.) S. Y. Kondr., Fedorenko, S. Stenroos, Kärnefelt, Elix, Hur et A. Thell - Romania, Suceava County, Călimani Mts, in the village Gura Haitii. Roadside trees along the river Neagra Şarului, on bark (Larix). Lat.: $47^{\circ} 12^{\prime} 10.1^{\prime \prime} \mathrm{N}$; Long.: $25^{\circ} 16^{\prime} 24.9^{\prime}$ E; Alt.: 1,073 m a.s.l. Leg.: Lőkös, L., 26.06.2009 [BP 93470].

! Melanelia bepatizon (Ach.) A. Thell - Romania, Călimani, Reservation Pinus Cembra, on rock. Lat.: $47^{\circ} 06.379^{\prime} \mathrm{N}$; Long.: $25^{\circ} 14.430^{\prime} \mathrm{E}$, Alt.: 1,650 m a.s.l. Leg.: Hur, J.-S. (RO-09097), 28.06.2009 [KoLRI 13047].

Melanelia stygia (L.) Essl. - M-ții Călimanului, vf. Pietrosul, 2,100 m alt., (creşte pe pietre silicioase). Coll.: Codoreanu, V., 07.1949 [CL as Parmelia stygia] (CodoreAnu 1952 as P. stygia, Moruzi et al. 1967 as P. stygia, Ciurchea 2004).

Melanobalea elegantula (Zahlbr.) O. Blanco, A. Crespo, Divakar, Essl., D. Hawksw. et Lumbsch - Rezervația Valea Repedea: izvorul pârâului 'Repedele' (STOIE and CRIŞAN 1998).

Melanohalea exasperata (De Not.) O. Blanco, A. Crespo, Divakar, Essl., D. Hawksw. et Lumbsch - Rezervaţia Pădurea din Şes (STOIE and CRIŞAN 1998 as Melanelia exasperata). - Romania, Suceava County, Călimani Mts, in the village Gura Haitii. Riverside trees along the river Neagra Şarului. On bark (Alnus). Lat.: $47^{\circ} 14^{\prime} 35.0^{\prime \prime} \mathrm{N}$; Long.: $25^{\circ} 14^{\prime} 28.8^{\prime \prime} \mathrm{E}$; Alt.: 1,026 m a.s.l. Leg.: Lőkös, L., 27.06.2009 [BP 93477]. - M-ții Călimani, Parcul Național Călimani, near Monastery 'Apa Rece', on bark, 27.06.2009 [hb. Crişan 2261].

Melanobalea exasperatula (Nyl.) O. Blanco, A. Crespo, Divakar, Essl., D. Hawksw. et Lumbsch - Rezervația Valea Repedea: izvorul pârâului 'Repedele' (STOIE and CRIŞAN 1998 as Melanelia exasperatula). - Romania, Suceava County, Călimani Mts, in the village Gura Haitii. Riverside trees along the river Neagra Şarului. On bark (Alnus). Lat.: $47^{\circ} 14^{\prime} 35.0^{\prime \prime} \mathrm{N}$; Long.: $25^{\circ} 14^{\prime} 28.8^{\prime \prime}$ E; Alt.: 1,026 m a.s.l. Leg.: Lőkös, L., 27.06.2009 [BP 93478].

Menegazzia terebrata (Hoffm.) A. Massal. - Rezervația Valea Repedea: pârâul 'Repedele', among mosses near the rock 'Deget', 21.07.1997 [hb. Crişan 1229] (STOIE and CRIŞAN 1998).

! Micarea lignaria (Ach.) Hedl. - Romania, Suceava County, Călimani Mts, Călimani National Park, Mt Rețitiş, near the meteorological station (Stația Meteo), ca $11 \mathrm{~km} \mathrm{SSW}$ of Gura Haitii, on acid soil. Lat.: $47^{\circ} 05^{\prime} 51.1^{\prime \prime} \mathrm{N}$; Long.: $25^{\circ}$ 14' 46.1" E; Alt.: 2,006 m a.s.1. Leg.: Lőkös, L., 25.06.2009 [BP 93337]. - Romania, Suceava County, Călimani Mts, near the village Gura Haitii in the valley of the stream Tarnița, on siliceous rock. Lat.: $47^{\circ} 11^{\prime} 22.8^{\prime}$ N; Long.: $25^{\circ} 14^{\prime} 17.9^{\prime \prime}$ E; Alt.: 1,102 m a.s.l. Leg.: Lőkös, L., 26.06.2009 [BP 93359]. - Romania, Suceava County, Călimani Mts, Călimani National Park, along tourist path between Stâncile Lucaciu and Stâncile Doisprezece Apostoli, on acid soil. Lat.: $47^{\circ} 13^{\prime} 27.9^{\prime \prime} \mathrm{N}$; Long.: $25^{\circ} 12^{\prime} 41.5^{\prime \prime}$ E; Alt.: 1,726 m a.s.l. Leg.: Lőkös, L., 27.06.2009 [BP 93493]. 
Montanelia disjuncta (Erichsen) Divakar, A. Crespo, Wedin et Essl. - Rezervația Bistricioru (STOIE 1999 as Melanelia disjuncta).

Nephroma resupinatum (L.) Ach. - Distr. Mureş: Călimani-Gebirge, in Valea Răstolniței, auf Buche (Servít and Cretzolu 1937, CretzoiU 1943a as N. resupinatum var. helvum, MORUZI et al. 1967, CIURCHEA 2004).

! Nephromopsis chlorophylla (Willd.) Divakar, A. Crespo et Lumbsch - Romania, Călimani, Potica (Gura Haitii), on bark. Lat.: $47^{\circ} 11.445^{\prime} \mathrm{N}$; Long.: $25^{\circ}$ 14.262' E, Alt.: 1,100 m a.s.l. Leg.: Hur, J.-S. (RO-09036), 26.06.2009 [KoLRI 12996 as Tuckermanopsis chlorophylla]. - Romania, Suceava County, Călimani Mts, Călimani National Park, Mt Negoi, near the sulphur mine (Exploatarea Călimani), on bark (Picea abies). Lat.: $47^{\circ} 06^{\prime} 24.7^{\prime \prime}$ N; Long.: $25^{\circ} 14$ ' 25.3” E; Alt.: 1,637 m a.s.l. Leg.: Lőkös, L., 28.06.2009 [BP 96309].

Nephromopsis cucullata (Bellardi) Divakar, A. Crespo et Lumbsch - Transsilvania: M. Kelemen-hegység, in andesiticis m. Magyar Negoj, alt. 1,900-2,000 m. Leg.: Jávorka, S. and Keller, J., 15.07.1941 [BP 96314] (Fig. 5). - M-ții Căli-

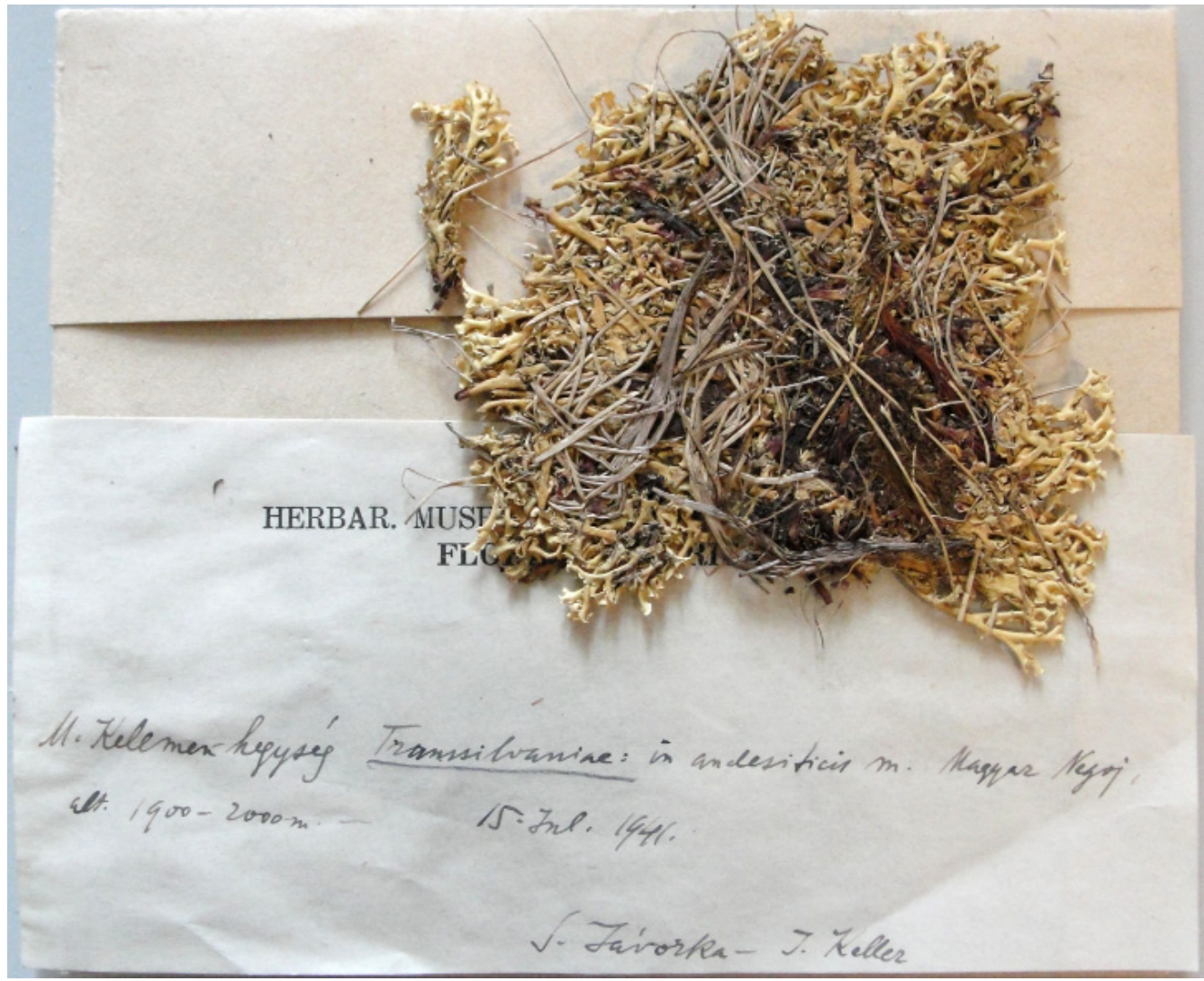

Fig. 5. Nephromopsis cucullata specimen collected by S. Jávorka and J. Keller at Magyar Negoj (Negoiul Unguresc) in 1941 (BP). 
manului, vf. Bradul Ciont, 1,700 m. alt. Coll.: Codoreanu, V., 07.1949 [CL as Cetraria cucullata] (Codoreanu 1952, Moruzi et al. 1967 as C. cucullata, Ciurchea 2004 as C. cucullata). - Romania, Călimani, Apostoli (Gura Haitii), on moss. Lat.: $47^{\circ} 13.643$ ' N; Long.: $25^{\circ} 12.840^{\prime}$ E, Alt.: 1,762 m a.s.l. Leg.: Hur, J.S. (RO-09070), 27.06.2009 [KoLRI 13026 as Flavocetraria cucullata]. - Romania, Călimani, Apostoli (Gura Haitii), on moss. Lat.: $47^{\circ} 13.453^{\prime}$ N; Long.: $25^{\circ} 12.715^{\prime}$ E, Alt.: 1,740 m a.s.l. Leg.: Hur, J.-S. (RO-09076), 27.06.2009 [KoLRI 13030 as F. cucullata]. - Romania, Suceava County, Călimani Mts, Călimani National Park, along tourist path between Stâncile Lucaciu and Stâncile Doisprezece Apostoli, on acid soil. Lat.: $47^{\circ} 13^{\prime} 27.9^{\prime \prime} \mathrm{N}$; Long.: $25^{\circ} 12^{\prime} 41.5^{\prime \prime} \mathrm{E}$; Alt.: $1,726 \mathrm{~m}$ a.s.l. Leg.: Lőkös, L., 27.06.2009 [BP 93494].

Nephromopsis nivalis (L.) Divakar, A. Crespo et Lumbsch - Distr. Mureş: munții Călimani, alt. 1,900-2,100 m s. m. Coll.: Nyárády, E. I. (Cretzoru 1939 as Cetraria nivalis, Moruzi et al. 1967 as C. nivalis, Ciurchea 2004 as C. nivalis). - M-ții Călimanului, vf. Bradul Ciont, circa 1,700 m alt. (Codoreanu 1952 as C. nivalis, Moruzi et al. 1967 as C. nivalis, CiurCheA 2004 as C. nivalis).

Ochrolechia parella (L.) A. Massal. - Distr. Mureş: munții Călimani pe vârful Tihu, pe roce eruptive, 1,700 m. s. m. (avec Haematomma ventosum, Ramalina carpathica et Ramalina strepsilis) (CRETzOIU 1943a as O. parella var. saxicola, Cretzoiu $1936 b$ as $O$. parella var. saxicola, Moruzi et al. 1967 as O. parella var. saxicola, Ciurchea 2004). - Romania, Călimani, Apostoli (Gura Haitii), on rock. Lat.: $47^{\circ} 13.453^{\prime} \mathrm{N}$; Long.: $25^{\circ} 12.715^{\prime}$ E, Alt.: $1,740 \mathrm{~m}$ a.s.l. Leg.: Hur, J.-S. (RO-09073), 27.06.2009 [KoLRI 13029]. - Romania, Suceava County, Călimani Mts, Călimani National Park, along tourist path between Stâncile Lucaciu and Stâncile Doisprezece Apostoli, on siliceous rock.. Lat.: $47^{\circ} 13^{\prime} 27.9^{\prime \prime} \mathrm{N}$; Long.: $25^{\circ}$ 12' 41.5” E; Alt.: 1,726 m a.s.l. Leg.: Lőkös, L., 27.06.2009 [BP 96290].

Ophioparma ventosa (L.) Norman - Distr. Mureş: Munții Călimani pe vârful Gruiu, 1,800 m. s. m., pe roce eruptive (CRETzolu 1943a as Haematomma ventosum, Moruzi et al. 1967 as H. ventosum, Ciurche a 2004). - M-ții Călimanului, vf. Bradul Ciont, 1,800 m. alt. Coll.: Codoreanu, V., 07.1949 [CL as H. ventosum] (CODOREANU 1952 as H. ventosum, Moruzi et al. 1967 as H. ventosum, Ciurchea 2004). - Romania, Călimani, Vf Rețitiş, on rock. Lat.: $47^{\circ} 05.847^{\prime} \mathrm{N}$; Long.: $25^{\circ}$ 14.737' E, Alt.: 2,022 m a.s.l. Leg.: Hur, J.-S. (RO-09024), 25.06.2009 [KoLRI 12984]. - Romania, Suceava County, Călimani Mts, Călimani National Park, Mt Rețitiş, near the meteorological station (Stația Meteo), ca $11 \mathrm{~km} \mathrm{SSW} \mathrm{of}$ Gura Haitii, on siliceous rock. Lat.: $47^{\circ} 05^{\prime}$ 51.1" N; Long.: $25^{\circ} 14^{\prime} 46.1^{\prime \prime}$ E; Alt.: 2,006 m a.s.l. Leg.: Lőkös, L., 25.06.2009 [BP 93343].

Parmelia omphalodes (L.) Ach. - Rezervația Valea Repedea: on soil and rock, among mosses near the rock 'Deget', 21.07.1997 [hb. Crişan 1239] (STOIE and CrişAN 1998). - Rezervaţia Bistricioru, Piatra Zurzugăului (STOIE 1999). 
Parmelia saxatilis (L.) Ach. - M-ții Călimanului, vf. Voivodesii, 1,800 m. alt. (pe scoarța arborilor şi pe roci). Coll.: Codoreanu, V., 28.07.1949 [CL 217870] (Codoreanu 1952, Moruzi et al. 1967, Ciurchea 2004). - Rezervația Valea Repedea: stânca 'Cubică'; stânca 'Deget' (STOIE and CRIşAN 1998). - Rezervația Bistricioru, pârâului Colbu (STOIE 1999). - Romania, Călimani, Apostoli (Gura Haitii), on rock. Lat.: $47^{\circ} 13.453^{\prime} \mathrm{N}$; Long.: $25^{\circ} 12.715^{\prime} \mathrm{E}$, Alt.: 1,740 m a.s.l. Leg.: Hur, J.-S. (RO-09074), 27.06.2009 [KoLRI 12784]. - Romania, Călimani, Potica (Gura Haitii), on bark. Lat.: $47^{\circ} 11.445^{\prime}$ N; Long.: $25^{\circ} 14.262^{\prime}$ E, Alt.: 1,100 m a.s.l. Leg.: Hur, J.-S. (RO-09038, RO-09039), 26.06.2009 [KoLRI 12998, KoLRI 12782]. - Romania, Călimani, Reservation Pinus Cembra, on rock. Lat.: $47^{\circ} 06.379^{\prime} \mathrm{N}$; Long.: $25^{\circ} 14.430^{\prime}$ E, Alt.: $1,650 \mathrm{~m}$ a.s.l. Leg.: Hur, J.-S. (RO-09096), 28.06.2009 [KoLRI 13046]. - Romania, Suceava County, Călimani Mts, Călimani National Park, Mt Rețitiş, near the meteorological station (Stația Meteo), ca $11 \mathrm{~km} \mathrm{SSW}$ of Gura Haitii, on siliceous rock. Lat.: $47^{\circ} 05^{\prime} 51.1^{\prime \prime} \mathrm{N}$; Long.: $25^{\circ}$ 14' 46.1” E; Alt.: 2,006 m a.s.l. Leg.: Lőkös, L., 25.06.2009 [BP 93344]. - M-ții Călimani, Parcul Național Călimani, near the meteorological station, on rock, 25.06.2009 [hb. Crişan 2212]. - Romania, Suceava County, Călimani Mts, Călimani National Park, along tourist path between Stâncile Lucaciu and Stâncile Doisprezece Apostoli, on siliceous rock. Lat.: $47^{\circ} 13^{\prime} 27.9^{\prime \prime} \mathrm{N}$; Long.: $25^{\circ}$ 12’ 41.5” E; Alt.: 1,726 m a.s.l. Leg.: Lőkös, L., 27.06.2009 [BP 96288].

Parmelia sulcata Taylor - Rezervația Pădurea din Şes, on trunks and twigs of oaks in the oak forest, 04.08.1996 [hb. Crişan 801] (STOIE and CRIŞAN 1998). Rezervația Valea Repedea: pârâul 'Repedele', stânca 'Cubică' (STOIE and CrişAN 1998). - Rezervația Bistricioru, Piatra Zurzugăului (Store 1999). - Romania, Călimani, Apostoli (Gura Haitii), on rock. Lat.: $47^{\circ} 13.453^{\prime}$ N; Long.: $25^{\circ} 12.715^{\prime}$ E, Alt.: 1,740 m a.s.l. Leg.: Hur, J.-S. (RO-09080), 27.06.2009 [KoLRI 12787].

Parmelina quercina (Willd.) Hale - Rezervația Pădurea din Şes, on oak twigs, 04.08.1996 [hb. Crişan 803] (STOIE and CrişAN 1998).

Parmelina tiliacea (Hoffm.) Hale - Rezervația Pădurea din Şes, on oak trunks in SE of the forest, 04.08.1996 [hb. Crişan 809] (STOIE and CRIŞAN 1998).

Parmeliopsis ambigua (Wulfen) Nyl. - Rezervația Bistricioru (Stole 1999). - Romania, Călimani, Potica (Gura Haitii), on bark. Lat.: $47^{\circ} 11.445^{\prime} \mathrm{N}$; Long.: $25^{\circ}$ 14.262' E, Alt.: 1,100 m a.s.l. Leg.: Hur, J.-S. (RO-09042), 26.06.2009 [KoLRI 13001].

Parmeliopsis hyperopta (Ach.) Vain. - Rezervația Bistricioru (STоIе 1999).

Parmotrema crinitum (Ach.) M. Choisy - Rezervația Valea Repedea: stâncii 'Deget', pârâul 'Scoruşet' (STOIE and CrişAN 1998).

Peltigera canina (L.) Willd. - M-ții Călimanului. Coll.: Codoreanu, V., 07.1949 [CL] Codoreanu 1952, Moruzi et al. 1967, Ciurchea 2004). - Rezervația Valea Repedea: on soil at the base of the rock 'Cubică', 04.08.1996 [hb. Crişan 811] (STOIE and CRIŞAN 1998). 
Peltigera didactyla (With.) J. R. Laundon - Distr. Mureş: munții Călimani, in valea Tihului, 1,500 m. s. m. (CRetzoiv 1943a as P. canina f. rufa, Moruzi et al. 1967 as P. spuria f. rufa, Ciurchea 2004). - M-ții Călimanului, Drâglele, $600 \mathrm{~m}$ alt. Coll.: Codoreanu, V., 07.1949 [CL 217846 as P. aphtosa] (CODOREANU 1952, Moruzi et al. 1967 as P. aphtosa, Ciurchea 2004 as P. aphtosa). - M-ții Călimanului, vf. Pietrosul, 2,000 m. alt. Coll.: Codoreanu, V., 07.1949 [CL as $P$. spuria] (CODOREANu 1952 as P. spuria, Moruzi et al. 1967 as P. spuria, CiURCHEA 2004).

Peltigera polydactylon (Neck.) Hoffm. - Rezervația Valea Repedea: on a stab on the waterfront of the stream 'Scoruşet', 09.08.1996 [hb. Crişan 702] (STOIE and CrişAN 1998).

! Peltigera praetextata (Sommerf.) Zopf - Romania, Călimani, Reservation Pinus Cembra, on moss. Lat.: $47^{\circ} 06.344^{\prime} \mathrm{N}$; Long.: $25^{\circ} 14.434^{\prime} \mathrm{E}$, Alt.: $1,655 \mathrm{~m}$ a.s.l. Leg.: Hur, J.-S. (RO-09094), 28.05.2009 [KoLRI 13044].

Peltigera rufescens (Weiss) Humb. - Distr. Mureş: munții Călimani, pe locuri ierboase in valea Tihului, 1,500 m. s. m.; vârful Pietrosul din Călimani, 2,100 m. s. m. şi în Poiana-Tihulet, pe sol (Cretzoiv 1943a, Moruzi et al. 1967, Ciurchea 2004). - Romania, Călimani, Tarnița (Gura Haitii), on rock. Lat.: $47^{\circ}$ 10.652' N; Long.: $25^{\circ} 13.139^{\prime}$ E, Alt.: 1,342 m a.s.l. Leg.: Hur, J.-S. (RO-09049), 26.06.2009 [KoLRI 13007]. - Romania, Călimani, Vf Negoi Romanesc, on soil. Lat.: $47^{\circ} 07.286^{\prime}$ N; Long.: $25^{\circ} 13.348^{\prime}$ E, Alt.: 1,680 m a.s.l. Leg.: Hur, J.-S. (RO09001), 25.06.2009 [KoLRI 12969].

Pertusaria albescens (Huds.) M. Choisy et Werner - Rezervația Pădurea din Şes, oak trunk in the northern part of the oak forest 04.08.1996 [hb. Crişan 783] (STOIE and CRIŞAN 1998).

Pertusaria amara (Ach.) Nyl. - M-ții Călimanului, Drâglele, 800 m. alt. (pe scoarță de copaci). Coll.: Codoreanu, V., 07.1949 [CL] (Codoreanu 1952, MoRUZI et al. 1967, CIURCHEA 2004).

Pertusaria corallina (L.) Arnold - Distr. Mureş: mts Călimani, sur Pietrosul, alt. 2,100 m., roches éruptives (CRETzOIU 1937 as $P$. corallina var. dolosa, 1943a as $P$. corallina var. dolosa, MoRUZI et al. 1967 as $P$. corallina var. dolosa, CiURCHEA 2004).

Pertusaria dactylina (Ach.) Nyl. - M-ții Călimanului, Bradul Ciont. Alt. cca. 1,800 m. Coll.: Codoreanu, V., 07.1949 [CL] (Codoreanu 1952, Moruzi et al. 1967, CiURCHEA 2004).

Pertusaria pertusa (L.) Tuck. - Distr. Mureş: Munții Călimani în valea Răstolnița pe Fagus (CRetzolu 1943a as P. pertusa var. polycarpa, Moruzi et al. 1967 as P. pertusa var. polycarpa, Ciurch EA 2004). - Distr. Mureş: mts Călimani sur le sommet Tihu, roches éruptives, alt. 1,750 m. (CRETzoiv 1937 as P. rupestris, $1943 a$ as $P$. rupestris, Moruzi et al. 1967 as $P$. rupestris, CiurChea 2004 as P. rupestris). 
Physcia dubia (Hoffm.) Lettau - Rezervaţia Pădurea din Şes, sunny trunks of isolated oaks, 04.08.1996 [hb. Crişan 787] (STOIE and CRIŞAN 1998).

Physcia stellaris (L.) Nyl. - Rezervaţia Pădurea din Şes, on young branch of oak, SW of oak forest, 04.08.1996 [hb. Crişan 791] (STOIE and CRIşAN 1998). - Romania, Suceava County, Călimani Mts, in the village Gura Haitii. Riverside trees along the river Neagra Şarului. On bark (Alnus). Lat.: $47^{\circ} 14^{\prime} 35.0^{\prime \prime} \mathrm{N}$; Long.: $25^{\circ} 14$ ' 28.8” E; Alt.: 1,026 m a.s.l. Leg.: Lőkös, L., 27.06.2009 [BP 93479].

Physcia tenella (Scop.) DC. - Rezervația Pădurea din Şes, on oak trunks, side with south exposure, 04.08.1996 [hb. Crişan 790] (STOIE and CRIŞAN 1998).

Physconia distorta (With.) J. R. Laundon - Rezervaţia Pădurea din Şes, on oak trunk, eastern part of the oak forest, 04.08 .1996 [hb. Crişan 788] (STOIE and CRIŞAN 1998).

Physconia muscigena (Ach.) Poelt - M-ții Călimanului, vf. Pietrosul, $2000 \mathrm{~m}$. alt. (pe sol). Coll.: Codoreanu, V., 07.1949 [CL as Physcia muscigena] (CodoreANU 1952 as P. muscigena, Moruzi et al. 1967 as P. muscigena, Ciurchea 2004).

! Placynthiella uliginosa (Schrad.) Coppins et P. James - Romania, Suceava County, Călimani Mts, Mt Negoi, near the sulphur mine (Exploatarea Călimani), ca $9 \mathrm{~km} \mathrm{SSW}$ of Gura Haitii, on acid soil. Lat.: $47^{\circ} 07^{\prime} 17.3^{\prime \prime} \mathrm{N}$; Long.: $25^{\circ} 13^{\prime}$ 20.0” E; Alt.: 1,673 m a.s.l. Leg.: Lőkös, L., 25.06.2009 [BP 93330]. - Romania, Suceava County, Călimani Mts, near Monastery at Cerbul, on acid soil. Lat.: $47^{\circ}$ 13' 57.4” N; Long.: 25 13' 35.4” E; Alt.: 1,618 m a.s.l. Leg.: Lőkös, L., 27.06.2009 [BP 93484].

Platismatia glauca (L.) W. L. Culb. et C. F. Culb. - Rezervația Valea Repedea (STOIE and CrişAN 1998). - Rezervaţia Bistricioru, pârâului Colbu (STOIE 1999). - Romania, Călimani, Potica (Gura Haitii), on bark. Lat.: $47^{\circ} 11.445^{\prime}$ N; Long.: $25^{\circ} 14.262^{\prime}$ E, Alt.: 1,100 m a.s.l. Leg.: Hur, J.-S. (RO-09041), 26.06.2009 [KoLRI 13000]. - Romania, Călimani, Vf Negoi Romanesc, on bark. Lat.: $47^{\circ} 07.286^{\prime} \mathrm{N}$; Long.: $25^{\circ} 13.348^{\prime}$ E, Alt.: 1,680 m a.s.l. Leg.: Hur, J.-S. (RO-09006, RO-09010), 25.06.2009 [KoLRI 12972, KoLRI 12976]. - M-ții Călimani, Parcul Național Călimani, Vf. Negoiul Românesc, on bark, 25.06.2009 [hb. Crişan 2220].

! Porpidia crustulata (Ach.) Hertel et Knoph - Romania, Suceava County, Călimani Mts, near the village Gura Haitii in the valley of the stream Tarnița, on siliceous rock. Lat.: $47^{\circ} 11^{\prime} 22.8^{\prime \prime} \mathrm{N}$; Long.: $25^{\circ} 14^{\prime} 17.9^{\prime \prime} \mathrm{E}$; Alt.: 1,102 m a.s.l. Leg.: Lőkös, L., 26.06.2009 [BP 93361].

! Porpidia macrocarpa (DC.) Hertel et A. J. Schwab - Romania, Suceava County, Călimani Mts, Călimani National Park, Mt Negoi, near the sulphur mine (Exploatarea Călimani), on decaying wood (Pinus cembra). Lat.: $47^{\circ} 06^{\prime} 24.7^{\prime \prime} \mathrm{N}$; Long.: 25 14’2 25.3” E; Alt.: 1,637 m a.s.l. Leg.: Lőkös, L., 28.06.2009 [BP 96300].

! Porpidia tuberculosa (Sm.) Hertel et Knoph - Romania, Suceava County, Călimani Mts, Călimani National Park, Mt Rețitiş, near the meteorological sta- 
tion (Staţia Meteo), ca $11 \mathrm{~km} \mathrm{SSW}$ of Gura Haitii, on siliceous rock. Lat.: $47^{\circ} 05^{\prime}$ 51.1" N; Long.: 25 14’ 46.1” E; Alt.: 2,006 m a.s.l. Leg.: Lőkös, L., 25.06.2009 [BP 93345].

Protopannaria pezizoides (Weber) P. M. Jørg. et S. Ekman - M-ții Călimanului, vf. Voivodesii, 1,800 m alt., (pe muşchi şi pe stânci). Coll.: Codoreanu, V., 07.1949 [CL as Pannaria brunnea] (CODOREANu 1952 as P. brunnea, Moruzi et al. 1967 as P. pezizoides, Ciurche 2004 as P. pezizoides). - Romania, Călimani, Tarnița (Gura Haitii), on rock. Lat.: $47^{\circ} 10.652^{\prime} \mathrm{N}$; Long.: $25^{\circ} 13.139^{\prime} \mathrm{E}$, Alt.: 1,342 m a.s.l. Leg.: Hur, J.-S. (RO-09050), 26.06.2009 [KoLRI 13008]. - Romania, Suceava County, Călimani Mts, in the valley of the stream Tarnița, on mossy soil. Lat.: $47^{\circ} 10^{\prime} 37.1^{\prime \prime} \mathrm{N}$; Long.: $25^{\circ} 13^{\prime}$ 08.3" E; Alt.: 1,345 m a.s.l. Leg.: Lőkös, L., 26.06.2009 [BP 93465].

Protoparmelia badia (Hoffm.) Hafellner - M-ții Călimanului, vf. Pietrosul. Coll.: Codoreanu, V., 07.1949 [CL as Lecanora badia var. cinerascens] (CODOREANU 1952 as L. badia var. cinerascens, MORUZI et al. 1967 as L. badia var. cinerascens, Ciurchea 2004). - Romania, Călimani, Vf Rețitiş, on rock. Lat.: $47^{\circ} 05.847^{\prime} \mathrm{N}$; Long.: $25^{\circ}$ 14.737' E, Alt.: 2,022 m a.s.l. Leg.: Hur, J.-S. (RO-09015), 25.06.2009 [KoLRI 12777]. - Romania, Suceava County, Călimani Mts, Călimani National Park, Mt Rețitiş, near the meteorological station (Stația Meteo), ca $11 \mathrm{~km} \mathrm{SSW}$ of Gura Haitii, on siliceous rock. Lat.: $47^{\circ} 05^{\prime} 51.1$ " N; Long.: $25^{\circ} 14^{\prime} 46.1$ ' E; Alt.: 2,006 m a.s.l. Leg.: Lőkös, L., 25.06.2009 [BP 93345]. - Romania, Suceava County, Călimani Mts, Călimani National Park, along tourist path between Stâncile Lucaciu and Stâncile Doisprezece Apostoli, on siliceous rock. Lat.: $47^{\circ} 13^{\prime} 27.9^{\prime \prime} \mathrm{N}$; Long.: $25^{\circ} 12^{\prime}$ 41.5” E; Alt.: 1,726 m a.s.l. Leg.: Lőkös, L., 27.06.2009 [BP 96289].

Protoparmeliopsismuralis (Schreb.) M. Choisy-M-ții Călimanului, vf. Bradul Ciont, 1,900 m. alt. Coll.: Codoreanu, V., 07.1949 [CL 217868 as Lecanora muralis var. albomarginata] (CODOREANU 1952 as L. muralis var. albomarginata, MORUZI et al. 1967 as L. muralis var. albomarginata, CiURCHEA 2004 as L. muralis).

Pseudephebe pubescens (L.) M. Choisy - M-ții Călimanului, vf. Pietrosul, 2,000 m alt., (creşte pe stânci silicioase). Coll.: Codoreanu, V., 07.1949 [CL as Parmelia pubescens] (CodoreANu 1952 as $P$. pubescens, Moruzi et al. 1967 as $P$. pubescens, Ciurchea 2004). - Romania, Călimani, Vf Rețitiş, on rock. Lat.: $47^{\circ}$ 05.847’ N; Long.: $25^{\circ} 14.737^{\prime}$ E, Alt.: 2,022 m a.s.l. Leg.: Hur, J.-S. (RO-09013), 25.06.2009 [KoLRI 12978].

Pseudevernia furfuracea (L.) Zopf - Munții Călimanului, (pe scoarța arborilor). Coll.: Codoreanu, V., 28.07.1949 [CL as Parmelia furfuracea] (CodoreANU 1952 as $P$. furfuracea, Moruzi et al. 1967 as $P$. furfuracea, CiurChea 2004). - Rezervația Pădurea din Şes \& Rezervația Valea Repedea (STOIE and CRIŞAN 1998). - Rezervația Bistricioru (Stole 1999). - Romania, Călimani, Vf Negoi Romanesc, on bark. Lat.: $47^{\circ} 07.286^{\prime} \mathrm{N}$; Long.: $25^{\circ} 13.348^{\prime} \mathrm{E}$, Alt.: 1,680 
m a.s.l. Leg.: Hur, J.-S. (RO-09005), 25.06.2009 [KoLRI 12775]. - Romania, Suceava County, Călimani Mts, Călimani National Park, Mt Rețitiş, near the meteorological station (Stația Meteo), ca $11 \mathrm{~km} \mathrm{SSW}$ of Gura Haitii, on bark (Pinus mugo). Lat.: $47^{\circ} 05^{\prime} 51.1^{\prime \prime} \mathrm{N}$; Long.: $25^{\circ} 14^{\prime} 46.1$ ' E; Alt.: 2,006 m a.s.l. Leg.: Lőkös, L., 25.06.2009 [BP 93351]. - Romania, Suceava County, Călimani Mts, Călimani National Park, along tourist path between Stâncile Lucaciu and Stâncile Doisprezece Apostoli, on acid soil. Lat.: $47^{\circ} 13^{\prime}$ 27.9' N; Long.: 25 $12^{\circ}$ 41.5” E; Alt.: 1,726 m a.s.l. Leg.: Lőkös, L., 27.06.2009 [BP 93495].

Psorinia conglomerata (Ach.) Gotth. Schneid. - M-ții Călimanului, vf. Voivodesii, 1,800 m. alt. Coll.: Codoreanu, V., 07.1949 [CL as Lecidea conglomerata] (CodoreANU 1952 as L. conglomerata Ach., Moruzi et al. 1967 as Toninia conglomerata, CIURCHEA 2004 as Biatora vernalis).

Pycnothelia papillaria (Ehrh.) L. M. Dufour - M-ții Călimanului, vf. Pietrosul, 2,050 m alt. Coll.: Codoreanu, V., 07.1949 [CL as Cladonia papillaria f. papillosa] (CODOREANu 1952 as C. papillaria f. papillosa, Moruzi et al. 1967 as C. papillaria f. papillosa, Ciurchea 2004).

Ramalina capitata (Ach.) Nyl. - Jud. Mureş: Munții Călimani, Vârful Tihu (Cretzoiv 1935 as R. strepsilis, Moruzi et al. 1967 as R. strepsilis, Ciurchea 2004).

Ramalina carpatica Körb. - Bezirk Mureş: Călimani-Gebirge, auf senkrechte felswände des Tihu-Gipfels, an Eruptivgestein, bei ca 1,700 m. s. m. (Cretzolu 1935, 1936a, Moruzi et al. 1967, CiURChEA 2004). - Romania, Călimani, Apostoli (Gura Haitii), on rock. Lat.: 47 $13.944^{\prime} \mathrm{N}$; Long.: $25^{\circ} 13.466^{\prime} \mathrm{E}$, Alt.: 1,732 m a.s.l. Leg.: Hur, J.-S. (RO-09066), 27.06.2009 [KoLRI 13024]. - Romania, Călimani, Apostoli (Gura Haitii), on rock. Lat.: $47^{\circ} 13.453^{\prime}$ N; Long.: $25^{\circ} 12.715^{\prime}$ E, Alt.: 1,740 m a.s.l. Leg.: Hur, J.-S. (RO-09075), 27.06.2009 [KoLRI 12785]. Romania, Suceava County, Călimani Mts, Călimani National Park, along tourist path between Stâncile Lucaciu and Stâncile Doisprezece Apostoli, on siliceous rock.. Lat.: $47^{\circ} 13^{\prime} 27.9^{\prime \prime} \mathrm{N}$; Long.: $25^{\circ} 12^{\prime} 41.5^{\prime \prime} \mathrm{E}$; Alt.: 1,726 m a.s.l. Leg.: Lőkös, L., 27.06.2009 [BP 96291].

Ramalina farinacea (L.) Ach. - M-ții Călimanului, valea Drâglelor, $600 \mathrm{~m}$ alt. (pe scoarța arborilor). Coll.: Codoreanu, V., 30.07.1949 [CL] (CodoreANU 1952, Moruzi et al. 1967, Ciurchea 2004). - Rezervația Pădurea din Şes, on shaded oak trunk, 05.08.1996 [hb. Crişan 799] (STOIE and CrişAN 1998). Rezervația Valea Repedea: pârâul 'Repedele' (STOIE and CRIŞAN 1998).

Ramalina fastigiata (Pers.) Ach. - M-ții Călimanului, valea Drâglelor. Coll.: Codoreanu, V., 30.07.1949 [CL as R. populina]. (CodoreAnU 1952 as R. populina, Moruzi et al. 1967, Ciurchea 2004).

Ramalina pollinaria (Westr.) Ach. - M-ții Călimanului, valea Drâglelor, 600 m. alt. Coll.: Codoreanu, V., 07.1949 [CL 217857] (Codoreanu 1952, 
Moruzi et al. 1967, Ciurchea 2004). - Rezervația Valea Repedea: on the rock 'Deget', 22.07.1997 [hb. Crişan 1301], stânca 'Cubică' (STOIE and CrişAN 1998).

! Rhizocarpon geographicum (L.) DC. - Romania, Călimani, Vf Rețitiş, on rock. Lat.: $47^{\circ} 05.847^{\prime} \mathrm{N}$; Long.: $25^{\circ} 14.737^{\prime} \mathrm{E}$, Alt.: 2,022 m a.s.1. Leg.: Hur, J.-S. (RO-09017), 25.06.2009 [KoLRI 12779]. - Romania, Suceava County, Călimani Mts, Călimani National Park, Mt Rețitiş, near the meteorological station (Staţia Meteo), ca $11 \mathrm{~km}$ SSW of Gura Haitii, on siliceous rock. Lat.: 47 $05^{\prime} 51.1^{\prime \prime} \mathrm{N}$; Long.: $25^{\circ} 14$ ' 46.1” E; Alt.: 2,006 m a.s.l. Leg.: Lőkös, L., 25.06.2009 [BP 93346].

Rbizocarpon reductum Th. Fr. - Distr. Mureş: mts Călimani, sur Pietrosul, alt. 2,100 m., roches éruptives (CRETZOIU 1937 as $R$. obscuratum f. fuscocinereum, SzatAl a 1942 as R. obscuratum f. fuscocinereum, MORUZI et al. 1967 as $R$. obscuratum f.fuscocinereum, CIURCHEA 2004 as $R$. obscuratum).

! Rinodina oxydata (A. Massal.) A. Massal. - Romania, Suceava County, Călimani Mts, near the village Gura Haitii in the valley of the stream Tarnița, growing together with Lecanora polytropa and Acarospora sp. on siliceous rock. Lat.: $47^{\circ} 11^{\prime} 22.8^{\prime \prime} \mathrm{N}$; Long.: $25^{\circ} 14^{\prime} 17.9^{\prime \prime}$ E; Alt.: 1,102 m a.s.1. Leg.: Lőkös, L., 26.06.2009 [BP 93362].

! Scoliciosporum chlorococcum (Stenh.) Vězda - Romania, Suceava County, Călimani Mts, in the village Gura Haitii. Riverside trees along the river Neagra Şarului. On bark (Alnus). Lat.: 47 14 ' 35.0” N; Long.: $25^{\circ} 14$ ' 28.8” E; Alt.: 1,026 m a.s.1. Leg.: Lőkös, L., 27.06.2009 [BP 93480].

Sphaerophorus fragilis (L.) Pers. - Distr. Mureş: munții Călimani pe vârful Pietrosul, 2,100 m. s. m. pe roce eruptive (CRETzOIU 1943a, MorUzi et al. 1967, Ciurchea 2004). - Romania, Călimani, Vf Rețitiş, on rock. Lat.: $47^{\circ} 05.847^{\prime} \mathrm{N}$; Long.: $25^{\circ} 14.737^{\prime}$ E, Alt.: 2,022 m a.s.l. Leg.: Hur, J.-S. (RO-09025), 25.06.2009 [KoLRI 12985]. - Romania, Suceava County, Călimani Mts, Călimani National Park, Mt Rețitiş, near the meteorological station (Stația Meteo), ca $11 \mathrm{~km} \mathrm{SSW} \mathrm{of}$ Gura Haitii, on acid soil. Lat.: $47^{\circ} 05^{\prime} 51.1^{\prime \prime} \mathrm{N}$; Long.: $25^{\circ} 14$ ' 46.1” E; Alt.: 2,006 m a.s.1. Leg.: Lőkös, L., 25.06.2009 [BP 93338].

Stereocaulon alpinum Laurer - M-ții Căliman. Alt. cca. 2,000 m. Coll.: Codoreanu, V., 28.07.1949 [CL]. - M-ții Călimanului, Vf. Voivodesii, 1,700 m. alt. Coll.: Codoreanu, V., 07.1949 [CL] (CodoreAnu 1952, Moruzi et al. 1967, Ciurchea 2004).

Stereocaulon paschale (L.) Hoffm. - Distr. Mureş: Munții Călimani, la Căliman-Izvor (Pietrosul-Ruschi, alt. 1,900-2,102 m s. m. Coll.: Nyárády, E. I., 22.07.1914 (Cretzoiu 1939, Moruzi et al. 1967, Ciurchea 2004). - Romania, Călimani, Vf Rețitiş, on rock. Lat.: $47^{\circ} 05.847^{\prime} \mathrm{N}$; Long.: $25^{\circ} 14.737^{\prime}$ E, Alt.: 2,022 m a.s.l. Leg.: Hur, J.-S. (RO-09023), 25.06.2009 [KoLRI 12983].

Stereocaulon vesuvianum Pers. - M-ții Călimanului, vf. Bradul Ciont, 1,850 m. alt. Coll.: Codoreanu, V., 29.07.1949 [CL as S. denudatum]. (CodoreAnu 
1952 as S. denudatum, Moruzi et al. 1967 as S. denudatum, Ciurchea 2004). Romania, Călimani, Vf Rețitiş, on rock. Lat.: $47^{\circ} 05.847^{\prime} \mathrm{N}$; Long.: $25^{\circ} 14.737^{\prime} \mathrm{E}$, Alt.: 2,022 m a.s.l. Leg.: Hur, J.-S. (RO-09030), 25.06.2009 [KoLRI 12990].

Tephromela atra (Huds.) Hafellner - M-ții Călimanului, vf. Pietrosul, 2,100 m. alt., (creşte pe pietre silicioase). Coll.: Codoreanu, V., 07.1949 [CL as Lecanora atra var. montana]. (CODOREANU 1952 as L. atra var. montana, MORUZI et al. 1967 as L. atra var. montana, CIURCHEA 2004).

Thamnolia vermicularis (Sw.) Schaer. - M-ții Călimanului, vf. Bradul Ciont, 1,800 m. alt., (creşte pe sol printre muşchi). Coll.: Codoreanu, V., 07.1949 [CL] (Codoreanu 1952, Moruzi et al. 1967, Ciurchea 2004). - Rezervația Bistricioru, Piatra Zurzugăului (SToıe 1999). - Romania, Călimani, Apostoli (Gura Haitii), on moss. Lat.: $47^{\circ} 13.643^{\prime} \mathrm{N}$; Long.: $25^{\circ} 12.840^{\prime} \mathrm{E}$, Alt.: $1,762 \mathrm{~m}$ a.s.l. Leg.: Hur, J.-S. (RO-09071), 27.06.2009 [KoLRI 13027]. - Romania, Călimani, Vf Rețitiş, on soil. Lat.: $47^{\circ} 05.847^{\prime} \mathrm{N}$; Long.: $25^{\circ} 14.737^{\prime} \mathrm{E}$, Alt.: $2,022 \mathrm{~m}$ a.s.l. Leg.: Hur, J.-S. (RO-09021), 25.06.2009 [KoLRI 12981]. - Romania, Suceava County, Călimani Mts, Călimani National Park, Mt Rețitiş, near the meteorological station (Stația Meteo), ca $11 \mathrm{~km} \mathrm{SSW}$ of Gura Haitii, on acid soil. Lat.: $47^{\circ}$ 05’ 51.1" N; Long.: 25 14’ 46.1" E; Alt.: 2,006 m a.s.l. Leg.: Lőkös, L., 25.06.2009 [BP 93340]. - M-ții Călimani, Parcul Național Călimani, Vf. Rețitiş, near the meteorological station, on soil, 25.06.2009 [hb. Crişan 2221].

Thelotrema lepadinum (Ach.) Ach. - Distr. Mureş: munții Călimani în valea Răstolnița, pe Conifere (Cretzoiv 1943a, Moruzi et al. 1967, CiurCheA 2004).

Toninia sedifolia (Scop.) Timdal - M-ții Călimanului, nord stâna Retițiş, 1,500 m. alt. Coll.: Codoreanu, V., 07.1949 [CL as T. coeruleonigricans var. subcandida] (CODOREANU 1952 as T. coeruleonigricans var. subcandida, MoRUZI et al. 1967 as T. coeruleonigricans var. subcandida, CIURCHEA 2004).

Trapeliopsis aeneofusca (Flot.) Coppins et P. James - M-ții Călimanului, mtele Puturosul, 1,250 m alt. (Codoreanu 1954 as Lecidea aeneofusca, Moruzi et al. 1967 as Lecidea aeneofusca, CiurcheA 2004).

! Tremolecia atrata (Ach.) Hertel - Romania, Suceava County, Călimani Mts, Călimani National Park, Mt Rețitiş, near the meteorological station (Stația Meteo), ca $11 \mathrm{~km} \mathrm{SSW}$ of Gura Haitii, on siliceous rock. Lat.: $47^{\circ} 05^{\prime} 51.1^{\prime \prime} \mathrm{N}$; Long.: $25^{\circ}$ 14’ 46.1” E; Alt.: 2,006 m a.s.1. Leg.: Lőkös, L., 25.06.2009 [BP 93348].

Umbilicaria crustulosa (Ach.) Lamy - Rezervația Valea Repedea: on the rock 'Cubică', 10.08.1996 [hb. Crişan 811] (STOIE and CRIŞAN 1998). - Romania, Călimani, Apostoli (Gura Haitii), on rock. Lat.: 47 13.964 ' N; Long.: $25^{\circ} 13.612^{\prime}$ E, Alt.: 1,751 m a.s.l. Leg.: Hur, J.-S. (RO-09061), 27.06.2009 [KoLRI 13019]. Romania, Suceava County, Călimani Mts, Călimani National Park, along tourist path between Stâncile Lucaciu and Stâncile Doisprezece Apostoli, on siliceous 
rock. Lat.: $47^{\circ} 13^{\prime} 27.9^{\prime \prime} \mathrm{N}$; Long.: $25^{\circ} 12^{\prime} 41.5^{\prime \prime} \mathrm{E}$; Alt.: 1,726 m a.s.l. Leg.: Lőkös, L., 27.06.2009 [BP 96293].

Umbilicaria cylindrica (L.) Delise - Distr. Mureş: munții Călimani pe vârful Pietrosul pe roce eruptive, 2,100 m. s. m., pe vârful Tihu şi in valea Tihului (Cretzolu 1943a as U. cylindrica var. typica, Moruzi et al. 1967 as U. cylindrica var. typica, Ciurchea 2004). - Distr. Mureş: munții Călimani pe vârful Vârful Gruiu, 1,800 m. s. m., pe roce eruptive (Cretzolu $1943 a$ as $U$. cylindrica f. microphylla, MORUzi et al. 1967 as U. cylindrica f. microphylla, CiURCHEA 2004). M-ții Călimanului. Coll.: Codoreanu, V., 07.1949 [CL as Gyrophora cylindrica] (Codoreanu 1952 as G. cylindrica), Moruzi et al. 1967, Ciurchea 2004). - Rezervația Valea Repedea: on the rock 'Cubică', 10.08.1996 [hb. Crişan 799] (STOIE and CRIŞAN 1998). - Rezervaţia Bistricioru (STOIE 1999). - Romania, Călimani, Apostoli (Gura Haitii), on rock. Lat.: 47 13.964 ' N; Long.: $25^{\circ} 13.612^{\prime}$ E, Alt.: 1,751 m a.s.l. Leg.: Hur, J.-S. (RO-09062), 27.06.2009 [KoLRI 13020]. Romania, Călimani, Apostoli (Gura Haitii), on rock. Lat.: $47^{\circ} 13.939^{\prime}$ N; Long.: $25^{\circ} 13.486$ ' E, Alt.: 1,729 m a.s.l. Leg.: Hur, J.-S. (RO-09064), 27.06.2009 [KoLRI 13022]. - Romania, Călimani, Apostoli (Gura Haitii), on rock. Lat.: $47^{\circ} 13.453^{\prime}$ N; Long.: $25^{\circ} 12.715^{\prime}$ E, Alt.: 1,740 m a.s.l. Leg.: Hur, J.-S. (RO-09072, RO-09082), 27.06.2009 [KoLRI 13028, KoLRI 13034]. - Romania, Călimani, Vf Rețitiş, on rock. Lat.: $47^{\circ} 05.847^{\prime} \mathrm{N}$; Long.: $25^{\circ} 14.737^{\prime} \mathrm{E}$, Alt.: $2,022 \mathrm{~m}$ a.s.l. Leg.: Hur, J.S. (RO-09012, RO-09018), 25.06.2009 [KoLRI 12977, KoLRI 12780]. - M-ţii Călimani, Parcul Național Călimani, Vf. Rețitiș, on rock, 25.06.2009 [hb. Crişan 2223]. - Romania, Suceava County, Călimani Mts, Călimani National Park, Mt Rețitiş, near the meteorological station (Stația Meteo), ca $11 \mathrm{~km} \mathrm{SSW}$ of Gura Haitii, on siliceous rock. Lat.: $47^{\circ} 05^{\prime} 51.1^{\prime \prime} \mathrm{N}$; Long.: $25^{\circ} 14^{\prime} 46.1^{\prime \prime} \mathrm{E}$; Alt.: 2,006 m a.s.l. Leg.: Lőkös, L., 25.06.2009 [BP 93349]. - Romania, Suceava County, Călimani Mts, Călimani National Park, along tourist path between Stâncile Lucaciu and Stâncile Doisprezece Apostoli, on siliceous rock. Lat.: $47^{\circ} 13^{\prime} 27.9^{\prime \prime} \mathrm{N}$; Long.: 25 12’ 41.5" E; Alt.: 1,726 m a.s.l. Leg.: Lőkös, L., 27.06.2009 [BP 96294].

Umbilicaria decussata (Vill.) Zahlbr. - Distr. Mureş: munții Călimani pe vârful Tihu, pe roce eruptive la 1,700 m. s. m. (CRETzolu $1943 a$ as U. cylindrica f. reticulata, Moruzi et al. 1967 as U. cylindrica f. reticulata, CiURCHEA 2004).

Umbilicaria deusta (L.) Baumg. - Rezervația Bistricioru, vârful Zurzugău spre cotul pârâului Colbu (Store 1999). - Romania, Călimani, Apostoli (Gura Haitii), on rock. Lat.: $47^{\circ} 13.964$ ' N; Long.: $25^{\circ} 13.612^{\prime} \mathrm{E}$, Alt.: $1,751 \mathrm{~m}$ a.s.l. Leg.: Hur, J.-S. (RO-09060), 27.06.2009 [KoLRI 13018]. - Romania, Călimani, Reservation Pinus Cembra, on rock. Lat.: $47^{\circ} 06.346^{\prime}$ N; Long.: $25^{\circ} 14.425^{\prime}$ E, Alt.: 1,660 m a.s.l. Leg.: Hur, J.-S. (RO-09093), 28.06.2009 [KoLRI 12789]. Romania, Hargitha County, Călimani Mts, Călimani National Park, near Lake Iezer, on siliceous rocks. Lat.: $47^{\circ} 05^{\prime} 33.6^{\prime \prime} \mathrm{N}$; Long.: $25^{\circ} 15^{\prime} 40.6^{\prime \prime} \mathrm{E}$; Alt.: $1,744 \mathrm{~m}$ 
a.s.l. Leg.: Lőkös, L., 25.06.2009 [BP 93356]. - Romania, Suceava County, Călimani Mts, Călimani National Park, along tourist path between Stâncile Lucaciu and Stâncile Doisprezece Apostoli, on siliceous rock. Lat.: $47^{\circ} 13^{\prime} 27.9^{\prime \prime} \mathrm{N}$; Long.: $25^{\circ} 12^{\prime} 41.5^{\prime \prime}$ E; Alt.: 1,726 m a.s.l. Leg.: Lőkös, L., 27.06.2009 [BP 96292]. - Romania, Suceava County, Călimani Mts, Călimani National Park, Mt Negoi, near the sulphur mine (Exploatarea Călimani), on siliceous rock. Lat.: $47^{\circ} 06^{\prime} 24.7^{\prime \prime} \mathrm{N}$; Long.: $25^{\circ}$ 14' 25.3" E; Alt.: 1,637 m a.s.l. Leg.: Lőkös, L., 28.06.2009 [BP 96312].

Umbilicaria spodochroa Hoffm. - M-ții Călimanului, vf. Bradul Ciont, 1,800 m. alt. Coll.: Codoreanu, V., 07.1949 [CL as Gyrophora cirrhosa] (CodoreANU 1952 as G. cirrhosa, Moruzi et al. 1967, CiUrChea 2004).

Umbilicaria vellea (L.) Ach. - Distr. Mureş: munții Călimani pe vârful Tihu şi pe Pietrosul, 2,100 m. s. m. pe roce eruptive (CREtzoiu 1943a, Moruzi et al. 1967, Ciurchea 2004).

Usnea cavernosa Tuck. - Distr. Mureş: mts Călimani, dans la Poiana-Tihulet, alt. 1,500 m., sur Picea excelsa (CRETzoiv 1937 as U. sibirica, MORUzi et al. 1967 as U. cavernosa subsp. sibirica, CiURCHEA 2004). - Rezervația Bistricioru, pârâului Colbu (Stoie 1999).

Usnea filipendula Stirt. - Rezervația Bistricioru (STOIE 1999). - Romania, Călimani, Apostoli (Gura Haitii), on bark. Lat.: 47 $13.453^{\prime}$ N; Long.: $25^{\circ} 12.715^{\prime}$ E, Alt.: 1,740 m a.s.l. Leg.: Hur, J.-S. (RO-09083, RO-09084), 27.05.2009 [KoLRI 13035, KoLRI 13036]. - Romania, Călimani, poteca (Gura Haitii), on bark. Lat.: $47^{\circ} 11.445^{\prime} \mathrm{N}$; Long.: $25^{\circ} 14.262^{\prime}$ E, Alt.: 1,100 m a.s.l. Leg.: Hur, J.-S. (RO09035), 25.06.2009 [KoLRI 12995].

Usnea florida (L.) F. H. Wigg. - M-ții Călimanului (CodoreAnU 1952, Moruzi et al. 1967, Ciurchea 2004). - Rezervația Valea Repedea: on dead spruce, left side of stream 'Ursului', 19.07.1997 [hb. Crişan 1182] (STOIE and CRIşAN 1998).

Usnea glabrata (Ach.) Vain. - Distr. Mureş: mts Călimani, sur Fagus silvatica (Cretzoiu 1937, 1941, 1943b, Moruzi et al. 1967, Ciurchea 2004).

Usnea birta (L.) F. H. Wigg. - Distr. Bistrița-Năsăud: Munții Călimani, pe Fagus. Leg.: M. Stoian. (Cretzoiu 1931, Moruzi et al. 1967, Ciurchea 2004).

Usnea lapponica Vain. - Jud. Mureş: Munții Călimani, Valea Răstolnita (Cretzoiv 1941 as U. substerilis, Cretzoiu $1943 b$ as U. substerilis, Moruzi et al. 1967 as U. substerilis, CiURCHEA 2004).

Usnea longissima Ach. - M-ții Călimanului, (pe Picea excelsa, Abies alba şi pe Fagus silvatica). Coll.: Codoreanu, V., 07.1949 [CL 204725] (Codoreanu 1952, Moruzi et al. 1967, CiURCheA 2004).

Usnea pendulina Motyka - M-ții Călimanului, Drâglele, 500 m. alt. (pe scoarță de Fagus sylvatica). Coll.: Codoreanu, V., 07.1949 [CL] (CodoreanU 1952, Moruzi et al. 1967, CiurChea 2004). 
Usnea subfloridana Stirt. - Distr. Mureş: munții Călimani, (pe Quercus petraea şi Picea excelsa) (Cretzolu 1937 as U. similis var. wainioi, Cretzolu 1941 as U. similis, Cretzoru $1943 b$ as U. similis var. wainioi, MorUZI et al. 1967 as $U$. comosa subsp. similis and as U. similis var. wainioi, CIURCHEA 2004 as U. subfloridana and as $U$. similis var. wainioi). - M-ții Călimanului, valea Drâglele, $700 \mathrm{~m}$ alt. (pe scoarță de copaci). Coll.: Codoreanu, V., 30.07.1949 [CL as U. comosa] (Codoreanu 1952 as U. comosa, Moruzi et al. 1967 as U. comosa, Ciurchea 2004). - Rezervația Valea Repedea: on old spruce twigs near an isolated rock on stream 'Repedele', 19.07.1997 [hb. Crişan 1187], pârâul 'Ursului' (STOIE and CRIŞAN 1998). - Rezervația Bistricioru, pârâului Colbu (STOIE 1999).

Varicellaria lactea (L.) I. Schmitt et Lumbsch - Distr. Mureş: munții Călimani pe vârful Tihu, 1,750 m. s. m., şi vârful Pietrosul, alt. ca. 2,100 m. s. m., pe roce eruptive (Cretzoiv 1943a as Pertusaria lactea, Moruzi et al. 1967 as P. lactea, Ciurchea 2004 as Ochrolechia lactea). - Distr. Mureş: munţii Călimani, vârful Pietrosul, alt. ca. 2,100 m. s. m. Coll.: Cretzoiu, P., 05.07.1935 [CL 441978 as P. lactea var. turocensis] (Fig. 6); pe vârful Gruiu, 2,100 m. s. m.; şi pe vârful Tihu, 1,750

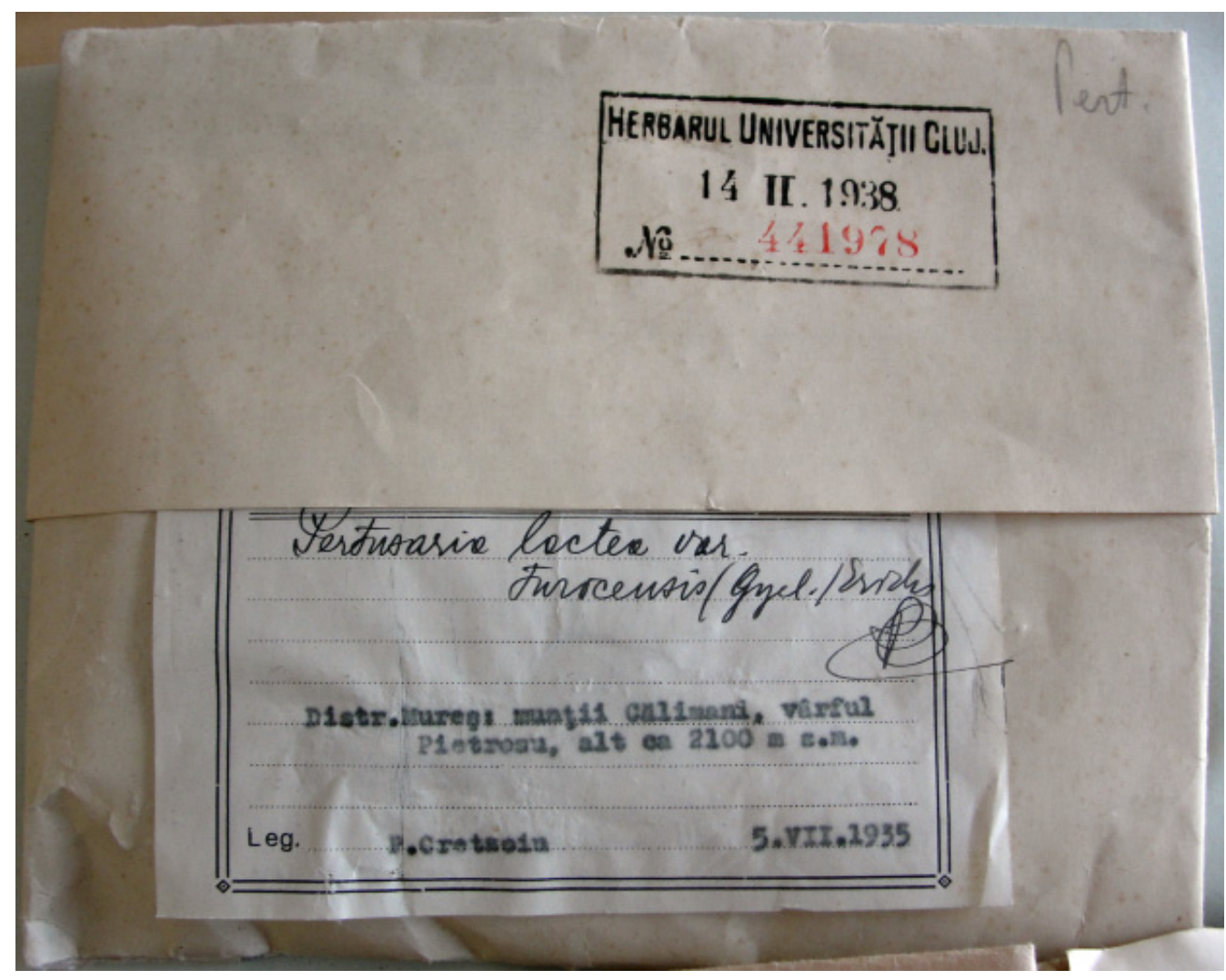

Fig. 6. Label of the Pertusaria lactea var. turocensis (= Varicellaria lactea) specimen collected by P. Cretzoiu in 1935 (CL 441978). 
m. s. m., pe roce eruptive (Cretzolu 1943a as P. lactea var. turocensis, MorUzi et al. 1967 as P. lactea var. turocensis, CiURChe A 2004 as Ochrolechia lactea). - M-ții Călimanului, Vf. Pietrosul, 2,000 m alt. (Codore Anu 1952 as Pertusaria lactea). Romania, Suceava County, Călimani Mts, near the village Gura Haitii in the valley of the stream Tarnița, on siliceous rock. Lat.: $47^{\circ} 11^{\prime} 22.8^{\prime \prime}$ N; Long.: $25^{\circ} 14$ ' 17.9” E; Alt.: 1,102 m a.s.l. Leg.: Lőkös, L., 26.06.2009 [BP 93363]. - Romania, Suceava County, Călimani Mts, Călimani National Park, along tourist path between Stâncile Lucaciu and Stâncile Doisprezece Apostoli, on siliceous rock. Lat.: $47^{\circ} 13^{\prime} 27.9^{\prime \prime} \mathrm{N}$; Long.: $25^{\circ} 12^{\prime} 41.5^{\prime \prime}$ E; Alt.: 1,726 m a.s.l. Leg.: Lőkös, L., 27.06.2009 [BP 96295].

Vulpicida pinastri (Scop.) J.-E. Mattsson et M.J. Lai - M-ții Călimanului, Vf. Pietrosul, 1,800 m alt., (pe scoarța coniferelor). Coll.: Codoreanu, V., 28.07.1949 [CL as Cetrariapinastri] (CodoreAnu 1952 as C.pinastri). - Rezervaţia Bistricioru (SToie 1999). - Romania, Călimani, Apostoli (Gura Haitii), on bark. Lat.: $47^{\circ}$ 14.473' N; Long.: $25^{\circ} 14.455^{\prime}$ E, Alt.: 1,637 m a.s.l. Leg.: Hur, J.-S. (RO-09053), 27.06.2009 [KoLRI 13011]. - Romania, Călimani, Potica (Gura Haitii), on bark. Lat.: $47^{\circ} 11.445^{\prime} \mathrm{N}$; Long.: $25^{\circ} 14.262^{\prime}$ E, Alt.: 1,100 m a.s.l. Leg.: Hur, J.-S. (RO09037), 26.06.2009 [KoLRI 12997]. - Romania, Suceava County, Călimani Mts, near Monastery at Cerbul, on bark (Picea abies). Lat.: $47^{\circ} 13^{\prime} 57.4^{\prime \prime} \mathrm{N}$; Long.: $25^{\circ}$ 13’ 35.4" E; Alt.: 1,618 m a.s.l. Leg.: Lőkös, L., 27.06.2009 [BP 93486].

Xanthoparmelia conspersa (Ach.) Hale - Rezervația Valea Repedea: on the horizontal side of the rock 'Cubică', 10.08.1996 [hb. Crişan 794] (STOIE and CRIŞAN 1998).

Xanthoparmelia mougeotii (D. Dietr.) Hale - Rezervația Bistricioru, Piatra Zurzugăului (STOIE 1999 as Parmelia mougeotii).

Xanthoria parietina (L.) Th. Fr. - Rezervaţia Pădurea din Şes, on sunny trunks of isolated oaks, SW side of oak forest, 04.08 .1996 [hb. Crişan 782] (STOIE and CRIŞAN 1998).

!! Xylographa pallens (Nyl.) Malmgren - Romania, Suceava County, Călimani Mts, Călimani National Park, Mt Negoi, near the sulphur mine (Exploatarea Călimani), on decaying wood (Pinus cembra). Lat.: $47^{\circ} 06^{\prime} 24.7^{\prime \prime} \mathrm{N}$; Long.: $25^{\circ} 14^{\prime}$ 25.3” E; Alt.: 1,637 m a.s.1. Leg.: Lőkös, L., 28.06.2009 [BP 96301] (Fig. 7).

! Xylopsora caradocensis (Nyl.) Bendiksby et Timdal - Romania, Suceava County, Călimani Mts, Călimani National Park, Mt Negoi, near the sulphur mine (Exploatarea Călimani), on bark (Pinus cembra). Lat.: $47^{\circ} 06^{\prime} 24.7^{\prime \prime}$ N; Long.: $25^{\circ}$ 14' 25.3” E; Alt.: 1,637 m a.s.l. Leg.: Lökös, L., 28.06.2009 [BP 96299].

\section{Excluded taxa}

The following taxa have been erroneously reported from the Călimani Mts by various literature sources. 


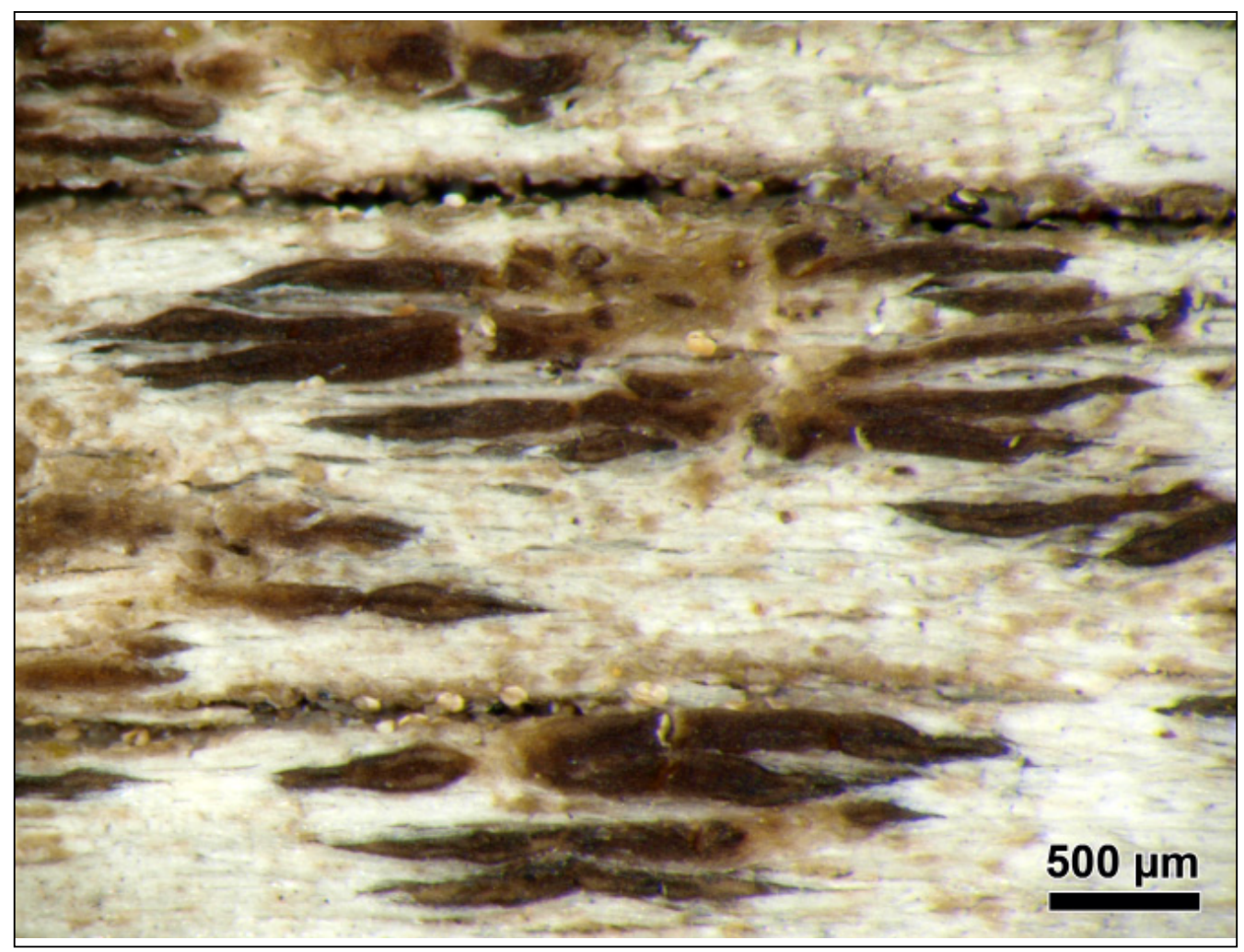

Fig. 7. Xylographa pallens from the recent collection from 2009 (BP 96301).

Nephroma endoxanthum Gyel. - Reg. Mureş A. M.: Mții Călimani, reg. Tuşnad, $800 \mathrm{~m}$ alt. (CodoreAnu 1954, Moruzi et al. 1967)

Peltigera degenii Gyeln. - Jud. Mureş: Munții Călimani, distr. Tuşnad, 800 m alt. (Codoreanu 1954 as P. virescens, Moruzi et al. 1967, Ciurchea 2004). Peltigera dolichorrbiza Nyl. - Jud. Mureş: Munţii Călimani, reg. Tuşnad, $700 \mathrm{~m}$ alt. (Codoreanu 1954, Moruzi et al. 1967, Ciurchea 2004).

\section{DISCUSSION}

Earlier collections from the Călimani Mts included mainly macrolichen species, e.g. from genera Cladonia (28), Parmelia (15), Umbilicaria (6), and Usnea (10). A total of 20 microlichen species were added recently from our new collection.

Altogether 170 taxa of lichen-forming and lichenicolous fungi are listed in this enumeration from various sources, 140 from published literature records and old herbarium specimens, and 30 species from recently collected material. One lichen species (Xylographa pallens), and two lichenicolous fungi (Clypeococcum 
hypocenomycis, Endococcus macrosporus) are new for Romania, and 30 lichen species are new for the Călimani Mountains. As a result of our short-time visit $38 \%$ of the old records can be confirmed by available voucher specimens with exact locality information and coordinates.

However, our knowledge of lichens and their lichenicolous fungi in the Călimani Mts is still limited. Further systematic and regular, fresh collections should be done to obtain new floristical results.

Acknowledgements - This work was supported by the National Research, Development and Innovation Fund (NKFI 119208, NKFI K 124341) and the Research Centre of Excellence 9878/2015/FEKUT; 11476-3/2016/FEKUT. We are grateful to Dr Katalin Bartók, Elena Cenuşa and Dan Grigoroaea for help in organising and guiding our fieldwork, to the directorate of the Călimani National Park for research permission, as well as to Dr Mihai Puşcaş, curator of the herbarium of the Alexandru Borza Botanical Garden, Babeş-Bolyai University (CL) in Cluj, Romania and to Ms Manuela Bojan, technician for their kind help in screening the lichen specimens of V. Codoreanu and P. Cretzoiu.

Összefoglaló: A Keleti-Kárpátokhoz tartozó Kelemen-hegység (Călimani Mts) zuzmóflórájáról viszonylag kevés információ áll rendelkezésre. 23 irodalmi forrás és mintegy 300 herbáriumi példány alapján összeállítottunk egy 170 taxonból álló listát, melyben összesítettük az értékelhető régi adatokat és a 2009-es gyűjtőutunk eredményeit. A Kelemen-hegység zuzmóflórájára 30 új (zömében mikrozuzmó-) fajt mutattunk ki, melyből a Xylographa pallens zuzmófaj, továbbá a Clypeococcum hypocenomycis és az Endococcus macrosporus zuzmóparazita mikrogombafajok egyben Románia flórájára is újak.

\section{REFERENCES}

Arup, U., Ekman, S., Lindblom, L. and Mattsson, J.-E. (1993): High performance thin layer chromatography (HPTLC), an improved technique for screening lichen substances. Lichenologist 25(1): 61-71. https://doi.org/10.1006/lich.1993.1018

BARTók, K. (1994): The family Stictaceae in Romania. - Contrib. Botanice, Cluj-Napoca 19931994: 47-57.

BARTó к, K. (1999): Genul Collema Weber ex Wigg. în România. - Studii şi cerc. Biol., Bistrița 5: 77-96.

BARTók, K. (2002): The lichen genus Chaenotheca (Th. Fr.) Th. Fr. in Romania. - Contrib. Botanice, Cluj-Napoca 37: 25-39.

BorHIDI, A. (1984): Role of mapping the fl ora of Europe in nature conservation. - Norrlinia 2: 87-98.

CABI (2018): The Index Fungorum. - http://www.indexfungorum.org (accessed 5 May 2018).

Ciurchea, M. (2004): Determinatorul lichenilor din România. - Editura BIT, Iaşi, 488 pp.

Codoreanu, V. (1952): Contribuțiuni la studiul florei lichenologice a Munților Căliman. (Contributions à l'étude de la flore lichénologique des montagnes Căliman). - Studii şi Cerc. şt. Acad. R.P.R. Filiala Cluj 1-2: 170-177. 
Codoreanu, V. (1954): Licheni noi şi rari pentru flora R.P.R. - Studii şi Cerc. şt., Acad. R.P.R. Filiala Cluj 3-4: 263-271.

Cretzolu, P. (1931): Contribuțiuni la flora lichenologică a României. - Bul. Soc. Studenților în Şt. Naturale, Bucureşti 2: 107-114.

Cretzolu, P. (1935): Ueber die geographische Verbreitung einiger Usneaceae aus Rumänien. Rev. Bryol. Lichénol. 8: 227-228.

Cretzoiu, P. (1936a): Lichenes Romaniae Exsiccati. Decas I. - Acta pro fauna et flora universali, Ser. II. Botanica, Bucureşti, 2(3): 7.

Cretzoiu, P. (1936b): Quelques lichens intéressants de Roumanie. I. - Rev. Bryol. Lichénol. 9: 139-142.

Cretzoiu, P. (1937): Quelques lichens intéressants de Roumanie. II. - Rev. Bryol. Lichénol. 10: $19-29$.

Cretzoiv, P. (1939): Lichenii colectati de E. I. Nyárády. - Bul. Grad. Bot. si Muz. Bot. Cluj 19(12): $104-108$.

Cretzolu, P. (1940): Lichenes Romaniae Exsiccati. Decas X (1940). - Acta pro fauna et flora universali, Ser. II. Botanica, Bucureşti.

Cretzoru, P. (1941): Flora lichenilor folioşi şi fruticuloşi epidendri şi epixili din România. - ICEF Referate-Comunicări, Ministerul Agriculturii şi Domeniilor, Bucureşti, Seria II, Nr. 47, 72 pp.

Cretzoiv, P. (1943a): Conspectul lichenilor Gymnocarpi din România, I. - Analele ICEF, Bucuresti, Ser. I. Partea II, 9: 1-222.

Cretzoiu, P. (1943b): Die Fundorte der bisher aus Rumänien bekanntgewordenen Arten der Gattung Usnea Wigg. - Bul. Politehnicii din Bucuresti 13(3-4): 400-411.

Cretzoiu, P. \& Klement, P. (1935): Stațiuni interesante de licheni din România. - Rev. Şt. V. Adamachi Iaşi 21(4): 206-207.

Moruzi, C., Petria, El. \& Mantu, El. (1967): Catalogul Lichenilor din România. - Acta Bot. Horti Bucurestiensis, 389 pp.

Niklfeld, H. (1971): Bericht über die Kartierung der Flora Mitteleuropa. - Taxon 20(4): 545571. http://dx.doi.org/10.2307/1218258

Orange, A., James, P. W. and White, F. J. (2010): Microchemical methods for the identification of lichens. 2nd ed. - British Lichen Society, London, $101 \mathrm{pp}$.

Robert, V., Stalpers, J. and Stegehuis, G. (2018): MycoBank, the fungal website. - http://www. mycobank.org/DefaultPage.aspx (accessed 5 May 2018).

Sérusiaux, E., Diederich, P., Brand, A. M. and Van den Boom, P. (1999): New or interesting lichens and lichenicolous fungi from Belgium and Luxembourg. VIII. - Lejeunia n.s. 162: $1-95$.

Servit, M. \& Cretzoiu, P. (1936): Flechten aus Rumänien, I. - Acta pro fauna et flora universali, Ser. II. Botanica, Bucureşti 2(2): 3-9.

Smith, C. W., Aptroot, A., Coppins, B. J., Fletcher, A., Gilbert, O. L., James, P. W. and Wolseley, P. A. (eds) (2009): The lichens of Great Britain and Ireland. - British Lichen Society, London, $1046 \mathrm{pp}$.

Stole, A. (1999): Studiul floristic asupra lichenilor foliacei şi fruticuloşi din rezervaţia Bistricioru (M. Călimani). - Studii şi cerc. Biol., Bistrița 5: 97-103.

STOIE, A. (2001): Analiza ecologică şi fitogeografică a lichenoflorei din rezervațiile „Bistricioru”, „Valea Repedea” şi „Pădurea din Şes”. (Ecological and phytogeographical analysis of the fruticose and foliose lichens from „Bistricioru”, „Valea Repedea” and „Pădurea din Şes” reserves. - Studii şi cerc. Biol., Bistrița 6: 71-80.

Stoie, A. \& CRişAN, F. (1998): Studiul floristic asupra lichenilor foliacei şi fruticuloşi din rezervațiile "Valea Repedea" şi "Pădurea din Şes" (Transilvania de Nord-Est). (Floristic studies on 
foliose and fruticose lichens from "Valea Repedea" and "Pădurea din Şes" reserves. - Studii şi cerc., Şt. Nat., Bistrița 4: 141-156.

Szatala, Ö. (1942): Lichenes Hungariae III. Gymnocarpeae (Cyclocarpineae: Peltigeraceae-Lecideaceae). - Folia Cryptog. II(5): 267-460.

Thiers, B. (2018, continuously updated): Index Herbariorum: a global directory of public herbaria and associated staff. - New York Botanical Garden's Virtual Herbarium. http://sweetgum. nybg.org/science/ih/.

Wirth, V., Hauck, M. and Schultz, M. (2013): Die Flechten Deutschlands. - Verlag Eugen Ulmer, Stuttgart, $1144 \mathrm{pp}$.

(submitted: 04.06.2018, accepted: 18.06.2018) 